Published in final edited form as:

Nat Rev Cancer. 2019 December ; 19(12): 716-732. doi:10.1038/s41568-019-0213-x.

\title{
Controversies around epithelial-mesenchymal plasticity in cancer metastasis
}

\author{
Elizabeth D. Williams ${ }^{1,2,3}$, Dingcheng Gao ${ }^{4}$, Andrew Redfern ${ }^{5}$, Erik W. Thompson ${ }^{1,2,{ }^{*}}$ \\ ${ }^{1}$ Institute of Health and Biomedical Innovation and School of Biomedical Sciences, Queensland \\ University of Technology (QUT), Brisbane, Queensland, Australia. \\ ${ }^{2}$ Translational Research Institute (TRI), Brisbane, Queensland, Australia. \\ ${ }^{3}$ Australian Prostate Cancer Research Centre -Queensland (APCRC-Q) and Queensland Bladder \\ Cancer Initiative (QBCI), Brisbane, Queensland, Australia. \\ ${ }^{4}$ Department of Cardiothoracic Surgery, Department of Cell and Developmental Biology and \\ Neuberger Berman Lung Cancer Center, Weill Cornell Medicine, New York, NY, USA. \\ ${ }^{5}$ Department of Medicine, School of Medicine, University of Western Australia, Fiona Stanley \\ Hospital Campus, Perth, Western Australia, Australia.
}

\section{Abstract}

\begin{abstract}
Experimental evidence accumulated over decades has implicated epithelial-mesenchymal plasticity (EMP), which collectively encompasses epithelial-mesenchymal transition and the reverse process of mesenchymal-epithelial transition, in tumour metastasis, cancer stem cell generation and maintenance, and therapeutic resistance. However, the dynamic nature of EMP processes, the apparent need to reverse mesenchymal changes for the development of macrometastases and the likelihood that only minor cancer cell subpopulations exhibit EMP at any one time have made such evidence difficult to accrue in the clinical setting. In this Perspectives article, we outline the existing preclinical and clinical evidence for EMP and reflect on recent controversies, including the failure of initial lineage-tracing experiments to confirm a major role for EMP in dissemination, and discuss accumulating data suggesting that epithelial features and/or a hybrid epithelial-mesenchymal phenotype are important in metastasis. We also highlight strategies to address the complexities of therapeutically targeting the EMP process that give consideration to its spatially and temporally divergent roles in metastasis, with the view that this will yield a potent and broad class of therapeutic agents.
\end{abstract}

Epithelial-mesenchymal transition (EMT) has well established roles in developmental programmes involved in generating new tissues and organs, and is followed, in most cases,

\footnotetext{
*e2.thompson@qut.edu.au.

Author contributions

All authors contributed equally to this manuscript.

Competing interests

The authors declare no competing interests.

Publisher's note

Springer Nature remains neutral with regard to jurisdictional claims in published maps and institutional affiliations.
} 
by the reverse process of mesenchymal-epithelial transition (MET) ${ }^{1-3}$. The EMT and MET processes also have instrumental roles in placentation ${ }^{4}$, endometrial function ${ }^{5}$ and fibrosis ${ }^{6}$. The dynamic combination of these processes is collectively encompassed by the term 'epithelial-mesenchymal plasticity' (EMP), which we and others advocate as a term of preference $^{7-13}$ over 'epithelial plasticity ${ }^{14,15}$, a more general term indicating flexibility in the epithelial state. By contrast, the terms EMT and MET are used to indicate the transitional directionality that is addressed in specific studies. The regulatory framework of EMP is well described, incorporating multiple pathways at numerous levels ${ }^{16,17}$. These processes are evolutionarily conserved with both common core elements and context-dependent molecular specializations in different species and in specific biological scenarios ${ }^{1,2}$. Moreover, EMP provides cells, tissues and organs with a range of mechanisms to influence growth and repair and handle diverse environmental stressors.

Cancer cells exploit EMP processes by manipulating a range of involved control mechanisms (FIG. 1). Consequently, EMP can then contribute directly or indirectly to several of the classical hallmarks of malignancy ${ }^{18,19}$, many of which manifest as an enhancement of the cancer stem cell (CSC) phenotype and increased metastatic potential $^{20-22}$. The core evidence supporting a role for EMP in metastasis stems from observations and functional evidence of the enhanced escape of mesenchymally shifted carcinoma cells from the primary tumour, together with their elevated survival, stemness and metastasis-initiation capacity relative to tumour cells with epithelial characteristics ${ }^{3}$. These observations are contrasted by evidence that experimental induction of enforced or stable mesenchymal features abrogate metastatic outgrowth in preclinical models, and that metastases display similar or enhanced epithelial properties relative to their primary tumours $^{22-25}$. Although much of the work on EMP in cancer focuses on carcinomas specifically, related plasticity programmes are described in other cancer types, including sarcomas ${ }^{26}$ and haematogenous tumours ${ }^{27}$. Changes in transcriptional programmes that are consistent with EMP have also been identified in stromal cells, likely contributing to the pathobiology of the tumour microenvironment ${ }^{28-30}$.

The role of EMP in cancer progression has not been universally accepted for multiple reasons, including the paucity of robust evidence for a process that is likely to be transient and episodic ${ }^{31,32}$, the relative scarcity of data supporting the occurrence of MET at the metastatic site, and observations that tumour cells can retain complete metastatic capability whilst maintaining an epithelial phenotype ${ }^{33-36}$. The literature is further confounded as several terms have been used to describe different aspects of the EMT process, including partial EMT (pEMT) and incomplete EMT, both of which refer to cells that have not fully transitioned along the epithelial-mesenchymal axis. We prefer to use the term pEMT, as this indicates that the positioning is deliberate with respect to the cell potentially harnessing beneficial properties from both polar states, whereas incomplete EMT implies that cells try to fully transition to a mesenchymal end point but are unable to do so. pEMT results in a hybrid state (with co-expression of both epithelial and mesenchymal markers in the same cell) or a metastable state (referring to the degree to which cells are fixed at their current position along the epithelial-mesenchymal axis $)^{37-40}$. Evidence from the past 2 years has shown that, among hybrid subpopulations, those that retain more epithelial features with less mesenchymal conversion have the greatest malignant and metastatic potential ${ }^{40,41}$. The 
relative expression of epithelial and mesenchymal markers might hypothetically be lower in these hybrid cells than in fully epithelial or mesenchymal cells, respectively, further challenging their identification in vivo and possibly compromising lineage-tracing efforts.

Elucidating the exact involvement of EMP in cancer progression, especially in metastasis, has been challenging, but therapies that target this novel and potentially unique cell population might demonstrate substantial anticancer efficacy with relatively low toxicity ${ }^{42}$. Furthermore, therapeutic control of EMP holds potential as a strategy by which to increase the sensitivity of tumours to a range of existing treatment modalities, including endocrine ${ }^{43}$, chemotherapeutic ${ }^{44}$, growth factor-targeted ${ }^{45}$, anti-angiogenic ${ }^{46}$ and radiotherapy-based strategies ${ }^{47}$ (FIG. 2). This approach likely extends to a broad spectrum of non-epithelial cancers, in which similar principles might apply under the banner of de-differentiation and re-differentiation.

Following a timely Viewpoint article addressing the knowledge gaps around EMP in cancer ${ }^{20}$, we emphasize herein the issues related to achieving clinical utility for EMP axis manipulation, with the aim of identifying the critical hurdles preventing its translation to improve patient outcomes. Accordingly, in this Perspectives article, we summarize the existing preclinical and clinical evidence for EMP in cancer, reflect on the controversies surrounding EMP, and describe therapeutic strategies to address the complexities of targeting a process with spatially and temporally divergent roles in metastasis.

\section{EMT and metastasis}

\section{Functional and clinical evidence}

Extensive in vitro evidence has delineated the contributions of EMT to tumour metastasis ${ }^{1,24}$, particularly in relation to tumour cell migration and invasion. In vivo studies also support a role for EMT in promoting metastasis, having correlated EMT status with the ability of malignant cells to escape the primary tumour. Genetically engineered mouse models (GEMMs) are an important preclinical resource that enable modelling of autochthonous cancers caused by known genetic drivers in an immuneintact syngeneic environment ${ }^{48}$ (BOX 1). Trimboli et al. ${ }^{49}$ surveyed a number of breast cancer GEMMs and found a high incidence of EMT events (25-64\% of tumours) in Myc oncogene-driven tumours, which model basal-like and claudin-low breast cancer, in contrast to the observation of infrequent EMT (0-5\% of tumours) in tumours from mouse mammary tumour virus (MMTV) promoter-driven polyoma middle T oncogene (MMTV-PyMT) and MMTV promoter-driven ERBB2 (MMTV-Neu) mice, which respectively model metastatic luminal breast cancer and HER2-amplified breast cancer. Interestingly, EMT frequency in the primary tumour, although dependent on the oncogenic driver, did not correlate with metastasis formation, as EMT ${ }^{\text {high }}$ tumours from MMTV promoter-driven MYC (MMTVMyc) mice did not give rise to lung metastasis, whereas EMT ${ }^{\text {low }}$ tumours from MMTVPyMT and MMTV-Neu mice reliably developed lung metastases ${ }^{49}$. Lineage-tracing in PTEN/p53-deficient and carcinogen-induced (medroxyprogesterone acetate (MPA) or 7,12dimethylbenz(a) anthracene (DMBA)) mouse mammary cancer models showed consistent evidence of pEMT tumour clones in the context of mostly epithelial tumour clones ${ }^{50}$. Consistent with this observation, in clinical tumours, advances in single-cell sequencing 
technology have revealed that, although pEMT is clearly evident in some cells, most tumour cells largely preserve the epithelial features seen in cells of their specific origin ${ }^{51}$. In our own preliminary clinical study in locally advanced breast cancers, the presence of EMT before neoadjuvant chemotherapy was associated with improved survival outcomes, whereas its presence after neoadjuvant chemotherapy was associated with poorer survival ${ }^{52}$, indicating that EMT does not automatically correlate with clinically significant metastasis. By contrast, patients with primary prostate, breast or lung cancers that exhibit the full range of EMP (that is, transcriptional evidence of both EMT and MET) have the poorest outcomes $^{12}$. The cellular context and the degree of EMT might explain these important differences, with considerable importance being attributed to the hybrid state ${ }^{42,53}$. In addition, the degree of plasticity and the extent of EMT seem to be important, with the highest metastatic potential having been demonstrated in tumour cell subpopulations retaining both epithelial and mesenchymal features whilst lacking the epithelial marker epithelial cell adhesion molecule (EpCAM) ${ }^{12,40}$.

Evidence for the prognostic importance of EMT in multiple carcinoma types abounds ${ }^{52}$ (TABLE 1), including associations between inferior patient outcomes and positive expression of mesenchymal markers (for example, vimentin, $\mathrm{N}$-cadherin, $\mathrm{a}$-smooth muscle actin (a-SMA) and S100A4) ${ }^{54,55}$, a lack of epithelial protein expression (for example, Ecadherin $^{56}$ and $\mathrm{EpCAM}^{57}$ ), expression of core EMT-activating transcription factors (EMTTFs; such as SNAIL1/2, ZEB1/2 and TWIST1/2) ${ }^{58}$ expression of epithelial-mesenchymal axis position stabilizers (for example, grainyhead-like protein 2 homologue (GRHL2) and Ovo-like 2 (OVOL2) $)^{59,60}$, and expression of microRNAs (miRNAs) that regulate these EMP processes (for example, miR-200 family members and miR-34) ${ }^{61}$. Specific core drivers of MET have also been described, such as carcinoembryonic antigen-related cell adhesion molecule 5 (CEACAM5; also known as CEA) ${ }^{62}$ and cellular communication network factor 6 (CCN6) ${ }^{63}$, although the relationship of these drivers with clinical outcome remains to be determined. In epithelial malignancies such as breast tumours, EMT is more prevalent in subtypes that are prone to distant dissemination such as basal-like ${ }^{55}$ and claudin-low ${ }^{64}$ malignancies. Consistent with this observation, tumours derived from tissues of mesenchymal origin are frequently highly invasive (for example, glioma) or characterized by early metastasis (for example, melanoma) ${ }^{65}$.

Beyond its direct contributions to tumour dissemination, EMP is also intertwined with the stemness properties of tumour cells, which confers abilities conducive to the successful expansion of metastatic deposits. Breast CSCs frequently exhibit EMT, the abrogation of which impinges on their stemness ${ }^{66-69}$. Although many examples support this relationship (reviewed in REF. ${ }^{70}$ ), the two processes are not inextricably linked and several studies demonstrate an uncoupling of stemness from the mesenchymal state. In particular, knockdown of $P R R X 1$, which encodes an EMT inducer, reversed EMT and promoted metastasis in breast cancer cells ${ }^{71}$; however, the carcinoma cells resulting from the MET retained elements of stemness. Furthermore, in human mammary epithelial (HMLER) cells, induction of a mesenchymal state by constitutive ectopic expression of ZEB1 led to loss of the epithelial-mesenchymal hybrid state (defined as CD104+/CD44 high in this study) and the corresponding adult stem cell programme, along with acquisition of enhanced tumorigenicity ${ }^{39}$. In summary, whilst EMT does not always correlate with metastasis and 
poor clinical outcomes, the hybrid state, in particular, has been implicated in a variety of carcinoma types. It will be critical to further refine approaches that enable the identification and quantification of tumour cells that are cycling through rounds of EMT and MET in clinical material to examine clinical utility.

\section{EMT timing and context}

Tumour cells might undergo EMT in the early stages of tumour initiation. Indeed, endogenous expression of EMT-TFs is observed in many normal tissues during pathological processes related to cancer pathogenesis. For example, EMT can be observed during carcinogenesis-accelerating inflammation, evidenced by the presence of EMT changes in normal colonic and pancreatic tissues during inflammatory bowel disease $\mathrm{e}^{72}$ and chronic pancreatitis ${ }^{73}$, both of which are associated with increased risk of subsequent malignancy. Similarly, EMT occurs during inflammation arising from chronic oral infections linked to oral carcinomas ${ }^{74}$ and in hepatitis B-driven inflammation associated with hepatocellular carcinoma $(\mathrm{HCC})^{75}$. Furthermore, carcinogens such as tobacco smoke and alcohol induce EMT in lung tissue ${ }^{76}$ and breast cells ${ }^{77}$, respectively.

EMT is also implicated in the development of malignancy through the suppression of oncogene-induced senescence ${ }^{78}$. Common pathological phenotypes of many tumours, such as the loss of a polarized single layer of epithelium, are consistent with EMT ${ }^{6}$. However, mutations in genes encoding EMT-TFs are not sufficient to drive ab initio tumorigenesis in mice, despite causing a range of developmental defects ${ }^{79-81}$. Rather, it is the pathological spatial and temporal regulation of EMT-TF expression that influences tumour progression.

EMT activation in tumour cells can be triggered by specific features of the local microenvironment in the rapidly growing primary tumour, such as hypoxia ${ }^{82-84}$, metabolic reprogramming ${ }^{85-90}$ and matrix stiffness ${ }^{91,92}$ (FIG. 1). Consequently, it is unsurprising that EMT is commonly observed at the tumour invasive front, where tumour cells directly interact with stromal cells and the extracellular matrix, or surrounding necrotic regions, where cells can be exposed to adverse microenvironmental conditions. Epithelial marker loss has been reported in budding tumour cells at the invasive front of primary colorectal cancers (CRCs), suggestive of EMT ${ }^{93-95}$, and further work has reported E-cadherin loss, increased nuclear ZEB1 staining and the acquisition of spindle cell morphology in these tumour buds ${ }^{94,96}$. Furthermore, a pEMT signature was characterized in a subset of head and neck squamous cell carcinomas (HNSCCs) using single-cell RNA-sequencing technology, particularly in cells at the invasive front of primary tumour nodules ${ }^{51}$. The pEMT tumour cells appeared to be directly in contact with cancer-associated fibroblasts (CAFs), with ligand-receptor analyses indicating the emission of pro-EMT signals from these stromal cells. Observations using intravital microscopy and lineage-tracing models for breast cancer have also provided direct evidence that a small population of primary tumour cells undergo EMT, often localized at the border area of tumour lobules adjacent to the vasculatureenriched stroma ${ }^{33,97}$. Similarly, tumour cell EMT is required to stimulate the appropriate metastatic niche, and this process is amplified via a positive feedback loop through which CAFs promote EMT $^{98}$. 
Heterogeneity in EMT status exists between tumour cells within a single primary tumour $^{51,99,100}$, and this spectrum of states has led to speculation that EMT programmes occurring later in cancer pathogenesis might be different from those involved in tumour initiation. In this context, many studies suggest that only a minor subpopulation ( 1-10\%) of the total primary tumour cell population harbours EMT events $33,34,100,101$. Although in vivo evidence from GEMMs did not support the hypothesis that the minority of EMT populations in the primary tumour were metastasis-initiating cells, it did not rule out the possibility of intercellular cooperation; thus, tumour cells that have undergone EMT could still have a key role in metastasis formation (BOX 2). In these contexts, cells that have undergone EMT might influence the behaviour of non-EMT neighbour cells, enabling them to utilize some of the benefits of the mesenchymal state without experiencing the drawbacks of EMT such as reduced proliferative capacity. Under hypoxic conditions, which commonly induce EMT ${ }^{51,82,84}$, exosomal transfer of WNT4 from CRC cells drove translocation of $\beta$ catenin to the nucleus in normoxic CRC cells, with a consequent increase in migration and invasion $^{102}$. In addition, extracellular vesicles from mesenchymally shifted 22RV1 human prostate cancer cells have been reported to induce mesenchymal changes in neighbouring cells through transforming growth factor- $\beta$ (TGF $\beta$ ) signalling and induce resistance to antiandrogens ${ }^{103}$, and exosomes from 30KT human bronchial epithelial cells following oncogene-induced EMT promoted chemoresistance in parental 30KT cells ${ }^{104}$.

Although much of the focus on the roles of EMP has been in advanced tumour scenarios, it has also been implicated in very early malignancy events, such that targeting EMT might have a broader application than first anticipated.

\section{EMT in circulating and disseminated tumour cells}

Analysis of circulating tumour cells (CTCs) can provide important insights into the molecular and cellular features of cancer cells in transit during the metastatic cascade. Observations of higher levels of mesenchymal gene expression in CTCs relative to tumour cells from both primary tumours and metastases provide a potential answer to the quandary that EMT is rarely observed in either primary tumours or metastases ${ }^{7,9,100}$. Indeed, RNA profiling of CTCs has shown a spectrum of states along the EMP axis ${ }^{100}$. In this seminal study, RNA in situ hybridization using pooled probes specific for epithelial or mesenchymal markers demonstrated a dynamic range of EMP states in breast cancer CTCs derived from an index patient, with shifts towards a predominantly mesenchymal state generally correlating with each episode of disease relapse ${ }^{100}$. In a study by Wu et al. ${ }^{105}$, classification of CTCs according to EMT marker expression in 12 patients with early-stage breast cancer and 6 patients with metastatic breast cancer revealed increased levels of mesenchymal CTCs as well as circulating tumour microemboli with a mesenchymal phenotype in metastatic cases Multi-marker assessment of early-stage breast cancer CTCs has also uncovered more frequent expression of mesenchymal markers (VIM, SNAI1 and UPAR) in lymph nodepositive patients compared with lymph node-negative patients ${ }^{106}$ and has been reported to provide prognostic information ${ }^{107}$.

Over the past 5 years, single-cell RNA-sequencing has been used to assess isolated CTC populations and CTC clusters ${ }^{108}$. Studies using xenografts of the LM2 variant of MDA- 
MB-231 human breast cancer cells have shown that CTC clusters are oligoclonal and have a more robust metastatic capacity than single CTCs, consistent with their association with poor prognosis in breast and prostate cancer, and have implicated the epithelial cell junction component plakoglobin (JUP) in CTC cluster formation ${ }^{109}$. Furthermore, a single-cell RNAsequencing study of CTCs in a mouse model of pancreatic cancer showed an overall loss of expression of the epithelial markers Chd1 (which encodes E-cadherin) and Muc1 (which encodes mucin-1) in CTCs compared with the primary xenograft tumours, with mixed and heterogeneous expression of mesenchymal markers in the same cells ${ }^{110}$. The authors also reported enriched expression of the pancreatic stem cell markers Aldh1a1 and Aldh1a2 in CTCs, but these events were not associated with an epithelial or mesenchymal state. Our group has seen enriched, but dysregulated, EMP (increases in both epithelial and mesenchymal gene expression) in CTCs compared with primary breast cancer xenograft tumours ${ }^{13}$. A further study reported enrichment for cell adhesion-related and proliferationrelated genes, along with the proliferative marker Ki-67, in CTC clusters compared with single CTCs from patients with breast cancer and from xenograft models ${ }^{111}$. In 2018, CTCs with CSC and pEMT phenotypes co-expressing cytokeratin, aldehyde dehydrogenase 1 (ALDH1) and nuclear TWIST1 $\left(\mathrm{CSC}^{+} / \mathrm{pEMT}^{+} \mathrm{CTCs}\right)$ were shown to have significant prognostic value in metastatic breast cancer compared with non-CSC ${ }^{+} / \mathrm{pEMT}^{+} \mathrm{CTCs}$, CTCs with $\mathrm{CSC}$ and epithelial phenotypes ( $\mathrm{CSC}^{+} /$epithelial-like $\mathrm{CTCs}$ ), or non-CSC${ }^{+} /$epitheliallike CTCs ${ }^{112}$. Furthermore, the incidence of $\mathrm{CSC}^{+} / \mathrm{pEMT}^{+} \mathrm{CTCs}$ was increased after firstline chemotherapy and, therefore, these might represent a chemoresistant subpopulation. In a breast cancer model, Padmanaban et al. ${ }^{36}$ showed that inducible knockdown of $C D H 1$ expression reduced the proliferation and survival of tumour cells as well as CTC numbers. Furthermore, our group has also reported reduced proliferation, both in vitro and in vivo, in MDA-MB-468 breast cancer cells after $C D H 1$ knockdown ${ }^{51}$. Consistently, in another study, EpCAM ${ }^{\text {low }}$ CTCs, whilst present in patients with breast and prostate cancer, lacked prognostic significance compared to their EpCAM ${ }^{\text {high }}$ counterparts ${ }^{113}$.

EMT is also frequently observed in disseminated tumour cells (DTCs) found in the bone marrow of patients with cancer ${ }^{7,10,114,115}$. This is an important observation, as a subpopulation of DTCs are believed to be precursors to macrometastases, but it is not yet possible to predict which specific cells will survive and escape dormancy to form a clinically significant tumour. The bone marrow is a relatively hypoxic tissue, raising the possibility that hypoxia-induced EMT processes in local stromal cells and arriving tumour cells promote the successful colonization of this niche followed by a period of dormancy ${ }^{116}$.

Given the position of CTCs and DTCs along the metastatic cascade, extensive data showing the enrichment of EMP in these cells compared with the tumours from which they originated is generally considered to be highly supportive of the role of EMP in metastasis. The observation that prognostic relationships are stronger for epithelial CTCs supports the notion that MET might be important prior to intravasation at the metastatic site, and might challenge the long-standing association between the mesenchymal phenotype and enhanced survival under adverse conditions. 


\section{MET and metastatic outgrowth}

\section{Epithelial characteristics in metastases}

The earliest indications of MET in the clinical setting were in colon adenocarcinomas, where cells at the invasive front were found to be mesenchymally shifted (as evidenced by nuclear $\beta$-catenin localization), whereas distal liver metastases were epithelial (as evidenced by cytoplasmic $\beta$-catenin localization) ${ }^{93}$. Subsequently, equal or stronger expression of the epithelial marker E-cadherin has been reported in distant metastases compared with matched primary breast and prostate cancer specimens ${ }^{117-121}$. Moreover, all metastatic tumours of invasive ductal carcinoma origin expressed E-cadherin, irrespective of the E-cadherin status of the primary breast tumours ${ }^{120}$. Similarly, E-cadherin expression has been seen in bone metastases originating from E-cadherin-negative, poorly differentiated primary breast carcinomas ${ }^{122}$. These observations are supported by transcriptional studies, in which metastases often cluster with their respective primaries ${ }^{123}$. In bladder cancer, the presence of EMT (loss of $C D H 1$ expression and elevated expression of mesenchymal markers $Z E B 1$, $Z E B 2$ and VIM) was associated with muscle-invasive bladder cancers but not with nonmuscle invasive bladder cancers ${ }^{124}$. However, among muscle-invasive bladder tumours, epithelial markers correlated with decreased survival, implying that mesenchymal and epithelial states are associated with different phases of tumour progression ${ }^{124}$.

These data collectively reinforce the epithelial requirements for metastases, which, when combined with the strong implications for mesenchymal capacity in earlier stages of the metastatic cascade, have led to the hypothesis regarding the requirement for MET following EMT.

\section{Metastatic capacity of epithelial variants}

A number of in vivo studies support a requisite role for EMT reversal in metastases. Using a series of T24 bladder cancer cell lines selected in vivo for increasing metastatic ability (via two rounds of intracardiac inoculation and outgrowth from resultant bone lesions, which is a relevant metastatic site), our group reported an association between macrometastatic competence and epithelial phenotype when cells were subsequently introduced either systemically or directly to bone ${ }^{125}$. We showed that the epithelial T24 bladder cancer variants (TSU-B1/B2) formed more metastases than the mesenchymal parental cells after intra-cardiac inoculation, albeit with a lower capacity to escape from the primary tumour when cells were implanted orthotopically. This observation supports the notion that mesenchymal attributes are important in initial escape from the primary tumour, whereas epithelial characteristics are required for the establishment of macrometastases. Consistent with this hypothesis, Celia-Terrassa et al. ${ }^{126}$ showed that stable mesenchymal variants of both T24 bladder cancer and PC-3 prostate cancer cell lines were unable to form metastases following intracardiac and orthotopic inoculation, respectively. Similarly, only cells with an epithelial phenotype (E-cadherin ${ }^{\text {high }}, \mathrm{CK} 18^{\text {high }}$ ) gave rise to lung and liver metastases following orthotopic inoculation in a panel of isogenic mouse breast cancer (4T1-derived) cell lines ${ }^{127}$. TWIST1-overexpressing breast cancer cells require E-cadherin for dissemination from organoids in vitro ${ }^{128}$, where they retain adherence junctions and proliferation ${ }^{129}$. Additionally, overexpression of miR-200 family miRNAs in the otherwise 
weakly metastatic $4 \mathrm{~T} 07$ cells increased metastasis from the orthotopic site ${ }^{130}$. Furthermore, a 2018 analysis implicated tightly balanced feedback loops producing bi-stability of cellular states, known as hysteresis, in control of metastasis ${ }^{131}$.

Manipulation of EMT signalling provides further evidence of the importance of MET in successful metastasis. The continuous activation of EMT signalling was shown to inhibit metastasis formation. Indeed, in an immunocompetent mouse breast cancer model (MMTVneuNT), mesenchymal tumour cells induced by SNAIL1 overexpression were unable to form macrometastatic lesions, although DTCs were readily observed ${ }^{132}$. Moreover, TWISTinduced EMT in squamous cell carcinoma cells promoted dissemination to the vasculature and distant organs in a mouse model, but TWIST had to be switched off for the formation of macrometastases $^{133}$. Similarly, loss of PRRX1-driven EMT was required for in vivo metastasis of BT-549 breast cancer cells despite the retention of stemness attributes throughout the process ${ }^{71}$. In another mouse model, induction of MET through overexpression of the transcription factor inhibitor of differentiation 1 (ID1) in HMLER cells promoted the conversion of micrometastases from TWIST-expressing HMLER tumours to macrometastases ${ }^{134}$. This was not seen with SNAIL1-expressing HMLER cells, consistent with the selectivity of ID1 in binding basic helix-loop-helix transcription factors.

Transient induction and reversal of EMT by inducible expression of SNAIL1 and SNAIL2 in prostate cancer cell lines has identified a specific MET-reversing gene signature that differed from the original epithelial transcriptional state ${ }^{12}$. This signature was enriched in clinical metastatic tissue, and its presence in primary prostate cancers was predictive of poor outcome, potentially due to an enhanced capacity of those primary tumours to undergo MET during metastasis. Interestingly, epithelial cells that had previously passed through the mesenchymal state were altered at the transcriptional level relative to cells permanently held in an epithelial state, showing enrichment of 'solid tumour', 'cell movement' and 'invasion of cells' gene sets as well as androgen and anti-androgen responsiveness. These findings imply that mesenchymal properties conducive to tumour spread and therapeutic resistance are retained. These data also highlight a level of EMT-MET cycling in the primary tumour, consistent with the observation of cells that have undergone EMT at the tumour invasive front in other studies ${ }^{33,93-97,135-137}$. Such cycling has been documented in a number of cancer types $^{11,138}$, and the incremental molecular impact has been revealed through both modelling and measurement ${ }^{131}$.

The collective observations of the epithelial nature of metastases and the failure of artificial EMT induction to promote tumour spread to the macrometastatic stage support the notion that MET has an important role in metastasis, at least in metastatic establishment at the secondary site, by enabling cohesive growth ${ }^{23,25}$. It is apparent that the incidence of MET likely varies depending on the metastatic site and is dependent on extrinsic microenvironmental signals received by the tumour cells at the secondary site. Along these lines, Pastushenko et al. ${ }^{40}$ showed that, although subcutaneously injected $\mathrm{EpCAM}^{-}$cells did not give rise to $\mathrm{EpCAM}^{+}$cells in secondary tumours, all $\mathrm{EpCAM}^{-}$subpopulations led to $\mathrm{EpCAM}^{+}$deposits when colonizing the lung microenvironment. In a mouse model, MDAMB-231 breast cancer cells required preconditioning of the MET-promoting microenvironment in the lung for metastasis formation, which led to upregulation of E- 
cadherin expression in MDA-MB-231 cells and the development of lung metastases ${ }^{139}$. In an experimental study, co-culture of DU145 prostate cancer cells with hepatocytes, modelling the respective metastatic niche in the liver, resulted in upregulation of $\mathrm{E}$ cadherin ${ }^{140}$. Such comparisons might need to be extended to bone, brain and/or liver metastases from the same patients or experimental animals, and could ultimately lead to selective tailoring of treatment of metastatic cancers based on their metastatic site. Aiello et al. ${ }^{141}$ found a shift from mesenchymal to epithelial histology as metastases in an autochthonous model of pancreatic ductal adenocarcinoma became larger. Furthermore, they also accumulated a desmoplastic stroma and became hypovascular, recapitulating their primary tumours.

The potential for MET has also been documented in cultured cell systems. Indeed, a dynamic flux between epithelial and mesenchymal states has been elegantly demonstrated in Dunning rat carcinoma cells ${ }^{11}$ and HCC-38 human breast cancer cells ${ }^{116}$. In addition, the PMC42-LA subline of the PMC42-ET human breast cancer cell line ${ }^{142}$ is stably epithelially shifted but shows dynamic plasticity. EMT marker expression is upregulated by epidermal growth factor (EGF) treatment in both lines, and sustained EMT induction in human mammary epithelial cells can become epigenetically stabilized ${ }^{143}$. Although the extent to which this inherent plasticity exists across cell lines is unclear, it does not seem to be universal, with only a minority of breast cancer cell lines showing such subpopulations ${ }^{138}$.

These studies further reinforce the requirement of epithelial features for metastatic competence across numerous models, despite the advantages associated with mesenchymal attributes in earlier stages of metastasis. A key point is the multitasking potential of cells exhibiting a hybrid phenotype, possibly explaining the otherwise heroic metastatic competence of epithelial variants despite major penalties in early steps such as migration, invasion, intravasation and survival as CTCs.

\section{EMP-independent metastasis}

Controversy regarding the role of EMP in metastasis stems from difficulties in generating evidence that cells exhibiting a predominantly epithelial phenotype in metastases actually underwent EMT ${ }^{34,144}$. Although EMT blockade by individually targeting crucial EMT-TFs does not necessarily impair metastasis, questions can be raised as to whether the right EMTTFs were targeted and/or whether other EMT-TFs compensated. In mice, genetic knockout of Snail1 or Twist 1 did not alter the emergence, dissemination or liver metastasis of invasive pancreatic cancer ${ }^{101}$, yet a similar approach of tissue-specific Zeb1 silencing resulted in a marked reduction in metastasis ${ }^{35,145}$. Moreover, breast tumour cell-specific knockout of Twist 1 impaired lung metastasis formation in a mouse model ${ }^{146}$. However, inhibiting EMT by overexpression of miR-200s, which directly target ZEB1 and ZEB2, did not impair lung metastasis formation in an orthotopic breast tumour GEMM ${ }^{34,147}$. By contrast, miR-200 levels in clinical breast tumours were positively associated with increased risk of metastatic spread $^{130}$. These studies support the requirement of MET for secondary tumour outgrowth and/or the dispensability of EMT for tumour cell escape from the primary site. Indeed, these authors showed a marked reduction in the number of CTCs in mice bearing miR-200transfected tumours, despite a dramatic stimulation of metastasis ${ }^{130}$. These inconsistent 
observations when targeting different EMT-TFs in different tumour models suggest a tumour-specific involvement of the EMT programme.

To precisely evaluate the contributions of EMT to tumour progression in vivo, lineagetracing strategies using GEMMs have been developed (BOX 1). Using novel reporters that integrate both transcriptional and post-transcriptional regulation to test whether MET is required for metastasis in cancer models, Somarelli et al. ${ }^{148}$ showed that metastasis of human uterine carcinosarcoma CS-99 cells from the subcutaneous site occurred via a METdependent pathway, whereas metastatic colonization appeared to be MET independent in two prostate carcinoma models (rat AT3 cells inoculated subcutaneously and human DU145 cells inoculated intravenously). Using pancreatic epithelium-specific Pdx1-Cre to mediate both oncogenic $K r a s^{\mathrm{G} 12 \mathrm{D}} / T p 53^{\text {flox/flox }}$ and yellow fluorescent protein (YFP) marker expression, EMT activations were observed in premalignant lesions, as evidenced by gain of ZEB1 and fibroblast-specific protein 1 (FSP1) expression and/or the loss of E-cadherin expression in tumour cells ${ }^{149}$. These EMT tumour cells $\left(\mathrm{YFP}^{+} / \mathrm{E}-\mathrm{cadherin}^{-}\right)$from the premalignant lesions exhibited enhanced tumorigenic capacity relative to their non-EMT $\left(\mathrm{YFP}^{+} / \mathrm{E}\right.$-cadherin $\left.{ }^{+}\right)$counterparts in vivo. However, tumour cells isolated from late-stage pancreatic tumours did not show such differential tumorigenic abilities. Both EMT and nonEMT cells gave rise to invasive pancreatic tumours and distant metastases regardless of their initial phenotypes when engrafted into recipient mice. Interestingly, tumours in both groups were histologically similar and contained a comparable prevalence of EMT events, suggestive of substantial EMP in these tumour cells ${ }^{149}$.

Given the EMP of tumour cells, it is challenging to demonstrate the direct contribution of EMT to metastasis in vivo. For this purpose, further lineage-tracing models have been developed using mesenchymal-specific promoters (for example, using the Fsp1 and Vim promoters) to mediate a fluorescent marker switch (from red fluorescent protein (RFP) to green fluorescent protein (GFP) expression) only in tumour cells that have undergone EMT $^{34}$ (BOX 1). These models enable the tracing of the EMT history of a cell with permanent markers that distinguish cells that revert to an epithelial phenotype after metastatic seeding from tumour cells that never underwent EMT. Unexpectedly, analysis of lung metastases from these breast cancer models did not demonstrate the presence of $\mathrm{GFP}^{+}$ cells. Rather, the metastases were chiefly composed of $\mathrm{RFP}^{+}$cells with an epithelial phenotype, indicating that they were derived from tumour cells that had not transitioned through EMT. By contrast, EMT events $\left(\mathrm{GFP}^{+}\right)$were detected in 1-2\% of primary tumour cells, which exhibited advantages in invasion, and were enriched among CTCs relative to primary tumour cells that were not marked as undergoing an EMT ${ }^{34}$. However, the primary tumour cells that had undergone EMT were vastly outnumbered by non-EMT-marked tumour cells; furthermore, these latter cells also resembled the majority of tumour cells in the metastatic outgrowth. It remains unclear how many cells that have undergone EMT would be necessary or sufficient to induce metastasis.

These findings evoked another round of vigorous debate about the real contributions of EMT in metastasis $1,24,147$. Specifically, concerns have been raised that certain mesenchymalspecific promoters might have limitations in their ability to fully describe the complex and diverse EMT process, that EMT lineage-tracing models might not be sensitive enough to 
report possible pEMT programming (given that, in this instance, both epithelial and mesenchymal features might be less strongly regulated relative to cells undergoing full EMT), and that cells with a strong enough EMT to activate the reporter system (which rarely occurs in the primary tumour but is enriched in CTCs) might not be able to reverse the EMT and subsequently colonize metastatic sites (BOX 1). Importantly, these studies did not deny the involvement of EMT in tumour progression, and EMT tumour cells clearly exhibited chemoresistant features that led to their contribution to metastasis after chemotherapy ${ }^{34,101}$. Thus, to fully understand the involvement of EMT in metastasis, novel and highly sensitive EMT lineage-tracing models that rely on multiple EMT markers and can report the dynamic change of phenotypes, ideally at the single-cell level, will be necessary.

One potential rationale for the requirement of MET in metastasis is the loss of proliferation observed with mesenchymal changes in many, but not all, systems. In support of this hypothesis, highly mesenchymal variants of T24 human bladder carcinoma cells and PC-3 human prostate cancer cells were unable to metastasize in immunocompromised mice as effectively as their more epithelial counterparts, and were compromised in their proliferative capacity $^{126}$. Our group has confirmed a loss of proliferative capacity in MDA-MB-468 human breast cancer cells in which E-cadherin expression has been supressed ${ }^{51}$, and this was also seen in two different mammary cancer GEMMs ${ }^{36}$. Furthermore, a 2018 study showed that IL-1 $\beta$-expressing innate immune cells that were systematically invoked by the primary tumour maintained metastasis-initiating cancer cells at the metastatic site in a ZEB1-positive differentiation state, preventing them from becoming proliferative Ecadherin-positive progeny ${ }^{150}$. Nevertheless, this relationship is not universal and examples of EMT systems that retain proliferative capacity have been reported ${ }^{151,152}$. However, ZEB1/2-driven EMT seems to be particularly associated with a decrease in proliferation ${ }^{153-155}$. Thus, the specific driver of EMT likely contributes to the variations in the apparent requirement for MET in the formation of metastases. Furthermore, the concomitant presence of independent oncogenic drivers of proliferation might override the otherwise inhibitory influence of EMT on proliferation.

\section{The hybrid state}

Hundreds of EMT-related genes have been characterized and evaluated to quantify the EMT status of cells and transcriptional metrics for quantification of EMT have been developed ${ }^{65,156}$. Among all predictors, the VIM:CHD1 gene expression ratio combined with the expression of CLDN7 (which encodes claudin 7) was shown to be the best approach for the assignment of various tumour cells into three phenotypes - epithelial, hybrid and mesenchymal ${ }^{156}$. Using this approach, metastatic breast tumours were categorized as either having an epithelial or hybrid phenotype. Another study evaluating EMT status - as defined by an alternate EMT quantification algorithm - showed that cell lines and cancers from different origins had different EMT phenotypic ranges, but the EMT status did not correlate with either tumour aggressiveness or poor survival outcomes in patients ${ }^{65}$. Segregation of the epithelial and mesenchymal components of this score showed that breast cancer specimens in The Cancer Genome Atlas were predominantly hybrid in nature, and that many of the mesenchymally classified breast cancer cell lines (basal B subgroup) also 
exhibited a high expression of both epithelial and mesenchymal markers and, therefore, also had a hybrid phenotype ${ }^{157}$.

With the exception of CTCs, the hybrid EMT state of human cancers has been mostly characterized using bulk tumour transcriptomes. Thus, the hybrid EMT status might be due to the contamination of stromal components ${ }^{158}$. Of course, the differential components in stroma might also provide independent clues to tumour subtypes ${ }^{159}$. In this instance, singlecell RNA sequencing results should be more informative, as it would enable distinction between mesenchymal stromal cells and EMT tumour cells. Indeed, a pEMT programme including upregulation of mesenchymal genes and genes encoding extracellular matrix proteins in conjunction with downregulation of certain epithelial genes was confirmed in a subset of HNSCC cells ${ }^{51}$. This programme did not include genes encoding the classical EMT-TFs SNAIL1, TWIST1/2 or ZEB1/2, although this finding could be due to the currently limited ability of this technique to detect lowly expressed genes, leading to an incomplete characterization of EMT status. Metastatic breast cancer cells with activation of an EMT-related stem cell programme have also been characterized at the single-cell level ${ }^{160}$. However, there was very limited overlap among the EMT markers identified in these two studies ${ }^{51,160}$. Nevertheless, whether the hybrid state represents a stable phenotype or is a snapshot of the dynamic and fluctuating EMT status of individual tumour cells remains an open question, although very new and preliminary (not yet peer reviewed) single-cell RNA sequencing data suggests a continuum ${ }^{161}$. In SCC9 human HNSCC cells, cultured pEMThigh and $\mathrm{pEMT}^{\mathrm{low}}$ subpopulations remained distinct at $4 \mathrm{~h}$ and $24 \mathrm{~h}$ after cell sorting, but became indistinguishable after 4 days of culture, with both cultures recapitulating the distribution of marker expression in unsorted SCC 9 cells ${ }^{51}$. Similarly, distinct subpopulations of HNSCC cells returned to the proportions seen in unsorted cells over time, although hybrid mesenchymal subpopulations were more plastic than either the most highly mesenchymal or epithelial subpopulations ${ }^{40}$. Similar reversion of an EpCAM ${ }^{\text {low }}$ subpopulation of MMTVPyMT $^{33}$ and PMC42-LA ${ }^{162}$ cells has also been reported, and mutually regulated epithelial $\left(\mathrm{EpCAM}^{+}\right)$and mesenchymal $\left(\mathrm{EpCAM}^{-}\right)$subpopulations of the HCC-38 human breast cancer cell line have been shown to revert to a tightly controlled epithelial to mesenchymal ratio $^{33}$. Moreover, relatively stable hybrid cell populations with greater tumorigenicity than epithelial or mesenchymal cells have been described. In these populations, the hybrid state was maintained by the EMT-TF SNAIL1 in concert with canonical WNT signalling, whereas constitutive ectopic expression of ZEB1 led to a fully mesenchymal shift, noncanonical WNT signalling, and loss of tumorigenicity in these cells $\mathrm{s}^{39}$.

A degree of finesse to EMT control has emerged with the identification of phenotypic stability factors (such as GRHL2 and OVOL2), which can interact with the standard EMT control machinery to stabilize the hybrid state $\mathrm{e}^{59,60}$. In the context of cancer, phenotypic stability factors have been shown to promote the dissemination of tumour cell clusters and their expression is associated with an inferior prognosis, whereas a fully mesenchymal signature correlated with improved survival outcomes ${ }^{163}$. Similarly, a bivalent chromatin configuration was identified in the promoter of the EMT-TF ZEB1 (REF. ${ }^{164}$ ), allowing rapid on-off cycling of ZEB1 expression in response to microenvironmental signals and control of tumorigenesis and/or outgrowth of secondary tumours. 
Collectively, the hybrid EMT state in tumour cells describes the presence of both epithelial and mesenchymal markers in the same tumour cells. It might reflect a stable state of tumour type or a transiting phase of tumour cells in switching their phenotype. Its correlation with aggressiveness and metastasis further enforces the crucial role of EMP in tumour progression.

\section{Therapeutic implications of EMP}

\section{Therapeutic resistance and EMT}

Highly pertinent to the clinical relevance of EMP is the relationship between therapeutic resistance and EMT, which has been well established in numerous studies ${ }^{70,165}$. Unlike the intertwined and sometimes conflicting literature associating EMT with the metastatic process, evidence supporting a role for EMT in treatment resistance is consistent and compelling. Enrichment of EMT markers at the RNA, protein and phenotypic levels has been described after exposure to a broad spectrum of therapeutic modalities, including hormonal therapies, chemotherapies, radiotherapy and many targeted therapies (FiG. 2). However, it is important to not simply confirm induction of EMT per se but to also to demonstrate its impact on treatment sensitivity, including in clinical cohorts.

In prostate cancer specimens, an EMT gene expression profile, which was only observed after neoadjuvant docetaxel and hormonal therapy, correlated with decreased time to relapse $^{44}$. Specifically, multivariate analysis revealed that low $C D H 1$ expression was predictive of reduced time to prostate-specific antigen relapse and high $Z E B 1$ expression correlated with rapid radiological progression. Furthermore, in prostate cancer, low tumour E-cadherin expression, which was mediated by reduced miR-200c and miR-205 expression, has additionally been linked to increased relapse rates after chemotherapy but not after surgery alone ${ }^{166}$. In breast cancer, EMT was enriched after docetaxel treatment ${ }^{167}$ and, in a second cohort, induction of EMT after chemotherapy correlated with increased expression of the ABC subfamily G member 2 (ABCG2) and ABC subfamily B member 1 (ABCB1; also known as MDR1) drug efflux proteins ${ }^{168}$; however, neither study reported patient outcomes. Our own study confirmed EMT induction after neoadjuvant chemotherapy in $24 \%$ of patients with breast cancer, which was associated with worse disease-free survival ${ }^{165}$. In another study, patients with late-stage breast cancer whose CTCs showed a pEMT phenotype (defined by expression of cytokeratin, ALDH1 and nuclear TWIST1) after chemotherapy had inferior progression-free survival, which was particularly marked in patients with HER2-negative cancers ${ }^{112}$. In patients with non-small-cell lung cancer who were treated with chemoradiotherapy, expression of mesenchymal markers in pretreatment specimens did not correlate with outcomes, but patients with evidence of EMT in their posttreatment resection specimen had inferior disease-free survival ${ }^{47}$. In patients with oesophageal cancer who had received chemotherapy, both increased SNAIL1 and decreased E-cadherin expression were predictive of poor chemotherapy response and inferior overall survival ${ }^{169}$. In patients with rectal cancer, reduced E-cadherin expression, nuclear $\beta$-catenin expression, reduced miR-200c expression and the presence of tumour budding were all associated with non-response to neoadjuvant chemoradiotherapy, whereas reduced expression of E-cadherin and miR-200c were both associated with reduced cancer-specific 
survival ${ }^{170}$. Beyond the aforementioned studies addressing the combination of chemotherapy and surgical treatments, radiotherapy has been shown to induce EMT in patients with CRC, which correlated with an increased recurrence rate ${ }^{171}$, and EMT-positive CTCs from patients with non-small-cell lung cancer have been shown to be enriched after radiotherapy 172,173 .

For endocrine treatment, despite extensive preclinical evidence for EMT as a driver of resistance to hormonal therapy, few clinical studies have been performed and have generally omitted direct survival correlations. In a breast cancer study, neoadjuvant letrozole induced EMT changes similar to the aforementioned chemotherapy-induced EMT, a finding that was confirmed in two independent cohorts; however, associations with patient outcomes were not explored $^{167}$. In further research into metastatic disease, SLUG (also known as SNAI2) expression in two independent cohorts of patients with oestrogen receptor-positive metastatic breast cancer treated with endocrine therapy was correlated with decreased progression-free survival ${ }^{174}$. There is also considerable preclinical evidence that androgen deprivation therapy, including luteinizing hormone-releasing hormone (LHRH) agonists and oral androgen receptor-binding anti-androgens, induce EMT in prostate cancer, leading to consequent anti-androgen resistance; however, clinical correlates are again lacking. For example, E-cadherin expression was decreased and expression of vimentin, $\mathrm{N}$-cadherin and ZEB1 were increased in clinical prostate cancer samples after development of insensitivity to combined androgen blockade relative to pretreatment specimens ${ }^{175}$. In a previously cited study ${ }^{44}$, combined androgen blockade and chemotherapy led to induction of EMT, which correlated with poor outcomes in early-stage prostate cancer.

A range of targeted therapies beyond endocrine blockade could also be affected, with evidence of EMT induction across multiple tumour types treated with agents against a wide spectrum of targets. In HER2-positive breast cancer cell lines, some clones underwent spontaneous EMT in culture, which led to trastuzumab resistance ${ }^{45}$, although the clinical significance has yet to be investigated. An EMT signature showed the strongest correlation with resistance to the EGF receptor inhibitor gefitinib in both lung and head and neck cancer cell lines ${ }^{176}$. Furthermore, EMT in $A L K$-translocated lung cancer cell lines led to resistance to the ALK inhibitor crizotinib ${ }^{177}$.

In addition to treatment-induced EMT, stimulation of EMT through various means prior to exposure to therapeutic agents has also been shown to make tumour cells less responsive to treatment relative to controls and could, therefore, have a clinical impact. Elements of the tumour microenvironment, such as hypoxia (which induces hypoxia-inducible factor-a (HIF1a) activity) ${ }^{178-181}$ and acidosis ${ }^{182,183}$, might attenuate chemo-responsiveness through EMT-related mechanisms. In keeping with these findings, induction of MET through inhibition of EMT-TFs can reverse therapeutic resistance (reviewed in REF. ${ }^{8}$ ) (FIG. 1).

Of additional interest, and intricately linked to the aforementioned EMT-associated therapeutic resistance, is the persistence of EMT or EMT-related changes that increase tumour aggressiveness after treatment. Beyond the impact of EMT in promoting resistance to the EMT-inducing treatment, EMT in tumours after therapy might result in increased proliferation, angiogenesis, immunosuppression and metastatic dissemination as well as 
suppression of apoptosis and induction of CSCs (reviewed in REF. ${ }^{165}$ ). Our own group has noted that, for virtually all therapies that have been established to induce EMT, eventual patient survival is shortened relative to the control period, implying accelerated progression, whereas for therapies known to reverse EMT, survival tends to be prolonged in excess of the duration of benefit from the treatment, implying that EMT reversal during treatment leads to less aggressive progression after treatment cessation ${ }^{165}$. Furthermore, considering the broad spectrum of cellular features affected by shifts along the epithelial-mesenchymal axis, it is unsurprising to find substantial changes in the parameters that govern the success of immunotherapy ${ }^{184,185}$ (BOX 3).

\section{Targeting EMP}

The involvement of EMT in the metastatic process, treatment resistance and accelerated progression post-therapy indicates that the ability to reverse or otherwise manipulate EMT could have therapeutic potential across a number of scenarios. The area warranting the greatest caution is that of direct EMT reversal with the aim of preventing metastatic spread, considering the aforementioned demonstration of the importance of the epithelial state in many contexts for the full establishment of macrometastases. In reality, the majority of treatment scenarios across the spectrum of cancer management involve directly treating, rather than preventing, existing metastases, be it micrometastases in the adjuvant setting or macrometastases in established disseminated malignancy. However, care is still required in the treatment of locally confined but unresectable cancers, lest dissemination be facilitated (FIG. 3). Careful context-specific experiments will be required to fully delineate the impact of such interventions on tumour pathogenesis, progression and dissemination.

In contrast to the dichotomous results regarding promotion of metastasis, the extensive preclinical data and generally supportive clinical data regarding the role of EMT in resistance to a broad spectrum of treatment modalities indicates the potential therapeutic value of EMT-reversing therapies in overcoming resistance and generates less concern regarding potential detrimental effects (FIG. 2). The many additional studies implicating EMT in accelerating progression after cessation of many such therapies strengthen this case. A wide range of agents that directly or indirectly target EMP processes have been reviewed elsewhere ${ }^{8,186}$. Considering this therapeutic potential, different approaches have been developed to manipulate the epithelial-mesenchymal axis, which can be classified into therapies that prevent or revert mesenchymal transition, target cells in the mesenchymal or hybrid states, inhibit transitions, fix cells to a position along the epithelial-mesenchymal axis, or target unique factors induced in cells after cycling through epithelial-mesenchymalepithelial states.

To date, the major initiatives have addressed the first therapeutic scenario - prevention or reversal of mesenchymal transition. In this space, a number of methods have been developed to harness various aspects of native cellular control of the EMT process. Non-coding miRNAs have established roles in the physiological repression of EMT (reviewed in REF. ${ }^{187}$ ); consequently, their use to prevent or reverse EMT is a rational consideration. The miR-200 family has emerged as having a fundamental place in suppression or reversal of EMT, with upregulation of miR-200s having been shown to render both chemoresistant 
prostate carcinoma cells ${ }^{166}$ and pancreatic cancer cells ${ }^{188,189}$ sensitive to chemotherapy. As an example of feasibility, clinical trials employing mimics of the tumour-suppressive miR-34 (REF. ${ }^{190}$ ) and miR-16 (REF. ${ }^{191}$ ) have now entered phase I clinical trials for the treatment of liver cancer and mesothelioma, respectively, with promising early results. The converse approach to delivery of such EMT-suppressive miRNAs is the therapeutic suppression of EMT-TFs. Extensive laboratory studies support the therapeutic potential of EMT-TF inhibition to increase treatment response across a range of therapeutic scenarios, including hormonal therapy for breast cancer ${ }^{192}$, chemotherapy for ovarian malignancy ${ }^{193}$ and targeted therapy for lung cancer ${ }^{194}$. Although EMT-TFs were previously considered undruggable due to a lack of functional domains targetable by small molecules, the rise of methods such as CRISPR-Cas9 and TALEN technology, which enable targeting of specific genes through genomic and epigenomic manipulation, has provided the tools to allow exploration of EMT-TF modulation; however, there are still considerable hurdles before clinical use is possible ${ }^{195-197}$. Modulation of upstream pathways that control or trigger EMT is also a compelling approach ${ }^{17,198,199}$, particularly as many pathways have either established agents or agents under development. In this regard, a number of systems have been successfully targeted to improve therapeutic activity using such concepts, including through targeting of $\mathrm{WNT}^{200}$, nuclear factor- $\mathrm{\kappa B} \mathrm{B}^{192}$ and ERK1/2 signalling ${ }^{201}$. Further consideration should be given to trials evaluating such therapeutic combinations with standard therapies across the treatment spectrum to harness the EMT-reversing properties of these approaches.

Beyond targeting tumour cells directly, there is mounting evidence that non-malignant stromal cells and non-cellular elements might drive EMT, implying that their manipulation could reverse the process. Indeed, evidence of this EMT-driving effect exists for tumourassociated macrophages ${ }^{202,203}$, neutrophils ${ }^{204}$, fibroblasts ${ }^{205}$, dense collagen ${ }^{206}$ and fibronectin ${ }^{207}$. As a proof-of-principal for the potential of targeting stromal factors, suppression of the M2 macrophage phenotype reversed EMT in tumour cells and resensitized lung tumours to paclitaxel in a human lung cancer cell line A549 xenograft mouse model ${ }^{208}$. In a co-culture model, antibody-mediated neutralization of neutrophilproduced IL-17a reversed neutrophil-induced EMT changes in gastric cancer cells and inhibited their migration and invasion ${ }^{204}$. Depletion of CAFs using a fibroblast-targeted immunotoxin inhibited TGF $\beta$ signalling, reduced tumour progression and enhanced chemosensitivity in the syngeneic $4 \mathrm{~T} 1$ breast cancer mouse model, which is consistent with inhibition of EMT, although the process was not directly assessed ${ }^{209}$. Furthermore, knockout of the gene encoding collagen VIII was reported to reduce EMT in renal cells in the context of experimental diabetic nephropathy ${ }^{210}$, although such studies have not been performed in cancer models.

Although many cytotoxic agents induce EMT, inhibitors of microtubule assembly, including eribulin and the vinca alkaloids, might exert the reverse effect (FiG. 2). Indeed, eribulin has been shown to reverse EMT in cancer cell lines and animal models ${ }^{211}$ and vinca alkaloids were reported to inhibit EMT-associated growth in lung cancer in vitro and in vivo ${ }^{212}$. Similarly, vinflunine reversed EMT and induced cell death in bladder cancer cell lines ${ }^{213}$. Hinting at this potential, phase III clinical trials of eribulin in both breast cancer and liposarcoma have demonstrated modest initial responses yet, unexpectedly, substantial 
survival benefits, which has led to the licencing of the drug for both indications ${ }^{214,215}$. Beyond single-agent use, these drugs could also be considered as part of combination regimens with chemotherapeutic agents known to drive EMT in the course of developing resistance.

The concept of transdifferentiation is related to the process of EMT reversal. During MET, malignant cells redifferentiate to reassume more of the characteristics of their epithelial cell of origin. During the transdifferentiation process, cells that have gained malignant potential through dedifferentiation to a mesenchymal form are driven along an alternative pathway involving differentiation to assume the properties of a different cell type. In a seminal example of this process, breast cancer cells that had undergone EMT were driven to differentiate into adipocytes using a combination of the peroxisome proliferator-activated receptor- $\gamma$ (PPAR $\gamma$ ) agonist rosiglitazone combined with the MEK inhibitor trametinib, losing the ability to invade and metastasize in the process ${ }^{216}$.

Regarding the therapeutic targeting of cells in the mesenchymal state, a high-throughput molecular screening approach was performed for agents with activity against mesenchymally shifted cancer lines, which identified three such agents — salinomycin, etoposide and abamectin ${ }^{217}$. Preclinical confirmation of ZEB1 downregulation and, consequently, chemosensitization of lymphoma cells in response to salinomycin treatment ${ }^{218}$, have led to the planning for clinical development of this agent. Following initial observations of improved survival rates among patients with breast cancer and comorbid diabetes who are being managed with metformin, this oral hypoglycaemic agent was subsequently found to reverse EMT and increase the sensitivity of chemoresistant breast cancer cells to chemotherapy ${ }^{219}$. To our knowledge, no initiatives have specifically targeted the partially transitioned cellular form, which might require further work to molecularly characterize key features of this hybrid state. However, the approach has conceptual merit.

Finally, arguably the most novel therapeutic concept is that of fixing cells at a given position on the epithelial-mesenchymal axis to prevent access to the range of states that might be required to facilitate different stages of the metastatic cascade. A mechanistic example of this process is the transcription factor OVOL, which interacts with the miR-200-ZEB1 regulatory axis to expand the hybrid epithelial-mesenchymal compartment and can influence the epithelial-mesenchymal axis bidirectionally ${ }^{220}$. If interventions could be developed to stabilize the hybrid state and restrict EMP, this approach could hypothetically prevent multiple elements of cancer progression.

\section{Conclusions and outstanding questions}

Controversies regarding the contribution of EMT to tumour progression stem primarily from the failure of some lineage-tracing experiments to identify cells in the metastatic site that have undergone EMT and, subsequently, MET ${ }^{34,101,147}$. By contrast, a plethora of data which are still accruing - have incriminated EMP in the metastatic process $35,71,133,144,145,148,149$. As described in this Perspectives article, there are possible technical reasons for these contrasting findings related to the sensitivity of detection of relevant epithelial-mesenchymal hybrid cells, which are also increasingly implicated in 
metastatic potential, whereby the lineage-tagging systems might not be strong enough to provide a readout of subtle changes in gene expression in an individual EMT target gene; accordingly, we await the development of more sensitive systems. At the same time, there are several studies that illustrate the arrival and establishment of carcinoma cells with epithelial traits at metastatic sites, which seems to confer maximal metastatic potential, at least in some scenarios, and might be necessary for macrometastatic outgrowth ${ }^{33,36,98,125,131}$. The degree to which these outwardly epithelial cells partially access elements of the mesenchymal phenotype during dissemination and whether an accentuating ability to shift between states would further increase the dissemination potential, remains to be determined.

In addition to these controversies, there are complexities arising from different interpretations of the EMT concept in various contexts. From primary tumour initiation to progression to metastasis formation, discrete forms of the EMT programme might be involved, such that considering the process as a single unvarying entity could be misleading. EMT is an enormous programme, with approximately one-third of the transcriptome regulated, and with both duplicity and diversity in the many mechanisms that regulate it, such that attempts to subclassify EMT forms might also illuminate current controversies between different experimental models. For example, EMT arising in tumours as a result of two distinct stimuli, EGF activation or hypoxia, was found to involve different effector pathways and behaviours and, importantly, resulted in differential changes in sensitivities to therapies ${ }^{221}$. Due to these substantial contextual differences, it is important to specify the EMT programme according to different tumour types, stages, local stressors and previous exposure to therapies.

EMT events in primary tumours are relatively rare. Nevertheless, certain EMT-related features could be involved in most tumour developmental steps. EMP bestows, on tumour cells, the ability to adapt to the changing microenvironment during secondary tumour formation and in the context of therapeutic treatment (FIGS 1,2), and might provide a unique opportunity for the development of new targeted therapies. Current therapeutic concepts mostly consider either the epithelial or mesenchymal phenotype, trying to target either state. This approach could limit the application of such therapies due to the ability of tumour cells to transition along the EMP axis to alternative non-sensitive states, and leads to additional considerations of assessing potential responders and therapeutic timing issues. However, targeting EMP to control the transitioning phase of tumour cells could restrict this route of escape and is, therefore, a promising direction for future research (FIG. 3). The detrimental impact of therapy-induced EMP adds breadth to the potential advantages that success in this endeavour could bring, adding reversal of resistance of established therapies to the achievable benefits. A combined therapy that blocks this avenue might, therefore, enhance the efficacy of conventional chemotherapies. Considering existing candidates, the EMT-reversal attributes of eribulin have been suggested to underpin its greater survival benefits in head-to-head comparisons with other therapies, relative to those promoting EMT $^{222}$. Expanding this concept, our own broader assessment of the survival benefits associated with a wide range of therapies confirms the disproportionately larger survival benefits for those therapies known to reverse EMT $^{39}$. 
In addition to targeting EMT processes in tumour cells directly, the manipulation of stromal cells implicated in contributing to EMT, such as CAFs or tumour-associated macrophages $98,209,223$, might represent another strategy. As they are not malignant cells, these targets have the advantage that clonal selection and acquisition of new mutations are not routes of resistance. Finally, having entered the era of efficacious immunotherapy, where vast resources are currently being committed to finding ways to extend the benefits of treatment from a modest proportion of patients to many, extensive evidence that EMT ties to immune suppression justifies efforts to expand our understanding of the effect of manipulating EMT in this context.

\section{Acknowledgements}

The authors acknowledge research support in the form of funding from the Australian Government Department of Health (Australian Prostate Cancer Centre -Queensland), Movember Foundation and the Prostate Cancer Foundation of Australia through a Movember Revolutionary Team Award (E.D.W.), Princess Alexandra Research Foundation (E.D.W, E.W.T.), National Breast Cancer Foundation (E.W.T.), the Australia India Council (E.W.T.), NIH (5R01CA205418; D.G.) and The Neuberger Berman Foundation Lung Cancer Research Center at Weill Cornell Medicine (D.G.). The Translational Research Institute receives funding from the Australian Government.

\section{References}

1. Nieto MA, Huang RY, Jackson RA \& Thiery JP EMT: 2016. Cell 166, $21-45$ (2016). [PubMed: 27368099]

2. Thiery JP, Acloque H, Huang RY \& Nieto MA Epithelial-mesenchymal transitions in development and disease. Cell 139, 871-890 (2009). [PubMed: 19945376]

3. Dongre A \& Weinberg RA New insights into the mechanisms of epithelial-mesenchymal transition and implications for cancer. Nat. Rev. Mol. Cell Biol. 20, 69-84 (2019). [PubMed: 30459476]

4. Yang X \& Meng T MicroRNA-431 affects trophoblast migration and invasion by targeting ZEB1 in preeclampsia. Gene 683, 225-232 (2019). [PubMed: 30315928]

5. Owusu-Akyaw A, Krishnamoorthy K, Goldsmith LT \& Morelli SS The role of mesenchymalepithelial transition in endometrial function. Hum. Reprod. Update 25, 114-133 (2019). [PubMed: 30407544]

6. Kalluri R \& Weinberg RA The basics of epithelial-mesenchymal transition. J. Clin. Invest. 119, 1420-1428 (2009). [PubMed: 19487818]

7. Alix-Panabieres C, Mader S \& Pantel K Epithelial-mesenchymal plasticity in circulating tumor cells. J. Mol. Med. 95, 133-142 (2017). [PubMed: 28013389]

8. Bhatia S, Monkman J, Toh AKL, Nagaraj SH \& Thompson EW Targeting epithelial-mesenchymal plasticity in cancer: clinical and preclinical advances in therapy and monitoring. Biochem. J. 474, 3269-3306 (2017). [PubMed: 28931648]

9. Francart ME et al. Epithelial-mesenchymal plasticity and circulating tumor cells: travel companions to metastases. Dev. Dyn. 247, 432-450 (2018). [PubMed: 28407379]

10. McInnes LM et al. Clinical implications of circulating tumor cells of breast cancer patients: role of epithelial-mesenchymal plasticity. Front. Oncol. 5, 42 (2015). [PubMed: 25767772]

11. Oltean $\mathrm{S}$ et al. Alternative inclusion of fibroblast growth factor receptor 2 exon IIIc in Dunning prostate tumors reveals unexpected epithelial mesenchymal plasticity. Proc. Natl Acad. Sci. USA 103, 14116-14121 (2006). [PubMed: 16963563]

12. Stylianou $\mathrm{N}$ et al. A molecular portrait of epithelial-mesenchymal plasticity in prostate cancer associated with clinical outcome. Oncogene 38, 913-934 (2019). [PubMed: 30194451]

13. Tachtsidis A et al. Human-specific RNA analysis shows uncoupled epithelial-mesenchymal plasticity in circulating and disseminated tumour cells from human breast cancer xenografts. Clin. Exp. Metastasis 36, 393-409 (2019). [PubMed: 31190270] 
14. Malek R, Wang H, Taparra K \& Tran PT Therapeutic targeting of epithelial plasticity programs: focus on the epithelial-mesenchymal transition. Cells Tissues Organs 203, 114-127 (2017). [PubMed: 28214899]

15. Santamaria PG, Moreno-Bueno G \& Cano A Contribution of epithelial plasticity to therapy resistance. J. Clin. Med. 8, 676 (2019).

16. Said NA, Simpson KJ \& Williams ED Strategies and challenges for systematically mapping biologically significant molecular pathways regulating carcinoma epithelial-mesenchymal transition. Cells Tissues Organs 197, 424-434 (2013). [PubMed: 23774256]

17. Thiery JP \& Sleeman J P Complex networks orchestrate epithelial-mesenchymal transitions. Nat. Rev. Mol. Cell Biol. 7, 131-142 (2006). [PubMed: 16493418]

18. Hanahan D \& Weinberg RA Hallmarks of cancer: the next generation. Cell 144, 646-674 (2011). [PubMed: 21376230]

19. Jolly MK et al. Implications of the hybrid epithelial/ mesenchymal phenotype in metastasis. Front. Oncol 5, 155 (2015). [PubMed: 26258068]

20. Brabletz T, Kalluri R, Nieto MA \& Weinberg RA EMT in cancer. Nat. Rev. Cancer 18, 128-134 (2018). [PubMed: 29326430]

21. Krebs MG et al. Molecular analysis of circulating tumour cells-biology and biomarkers. Nat. Rev. Clin. Oncol. 11, 129-144 (2014). [PubMed: 24445517]

22. van Denderen BJ \& Thompson EW Cancer: the to and fro of tumour spread. Nature 493, 487-488 (2013). [PubMed: 23344357]

23. Brabletz T EMT and MET in metastasis: where are the cancer stem cells? Cancer Cell 22, 699-701 (2012). [PubMed: 23238008]

24. Chaffer CL, San Juan BP, Lim E \& Weinberg RA EMT, cell plasticity and metastasis. Cancer Metastasis Rev. 35, 645-654 (2016). [PubMed: 27878502]

25. Thompson EW \& Haviv I The social aspects of EMT-MET plasticity. Nat. Med. 17, 1048-1049 (2011). [PubMed: 21900919]

26. Kahlert UD, Joseph JV \& Kruyt FAE. EMT-and MET-related processes in nonepithelial tumors: importance for disease progression, prognosis, and therapeutic opportunities. Mol. Oncol 11, 860877 (2017) [PubMed: 28556516]

27. Carmichael CL \& Haigh JJ The Snail family in normal and malignant haematopoiesis. Cells Tissues Organs 203, 82-98 (2017). [PubMed: 28214876]

28. Alba-Castellon L et al. Snail1 expression is required for sarcomagenesis. Neoplasia 16, 413-421 (2014). [PubMed: 24947186]

29. Baulida J Epithelial-to-mesenchymal transition transcription factors in cancer-associated fibroblasts. Mol. Oncol. 11, 847-859 (2017). [PubMed: 28544627]

30. Rowe RG et al. Mesenchymal cells reactivate Snail1 expression to drive three-dimensional invasion programs. J. Cell Biol. 184, 399-408 (2009). [PubMed: 19188491]

31. Christiansen JJ \& Rajasekaran AK Reassessing epithelial to mesenchymal transition as a prerequisite for carcinoma invasion and metastasis. Cancer Res. 66, 8319-8326 (2006). [PubMed: 16951136]

32. Thompson EW, Newgreen DF \& Tarin D Carcinoma invasion and metastasis: a role for epithelialmesenchymal transition? Cancer Res. 65, 5991-5995 (2005). [PubMed: 16024595]

33. Beerling E et al. Plasticity between epithelial and mesenchymal states unlinks EMT from metastasisenhancing stem cell capacity. Cell Rep. 14, 2281-2288 (2016). [PubMed: 26947068]

34. Fischer KR et al. Epithelial-to-mesenchymal transition is not required for lung metastasis but contributes to chemoresistance. Nature 527, 472-476 (2015). [PubMed: 26560033]

35. Krebs AM et al. The EMT-activator Zeb1 is a key factor for cell plasticity and promotes metastasis in pancreatic cancer. Nat. Cell Biol. 19, 518-529 (2017) [PubMed: 28414315]

36. Padmanaban $\mathrm{V}$ et al. E-cadherin is required for metastasis in multiple models of breast cancer. Nature 573, 439-444 (2019). [PubMed: 31485072]

37. Jia D et al. Testing the gene expression classification of the EMT spectrum. Phys. Biol. 16, 025002 (2019). [PubMed: 30557866] 
38. Klymkowsky MW \& Savagner P Epithelial-mesenchymal transition: a cancer researcher's conceptual friend and foe. Am. J. Pathol. 174, 1588-1593 (2009). [PubMed: 19342369]

39. Kroger $\mathrm{C}$ et al. Acquisition of a hybrid $\mathrm{E} / \mathrm{M}$ state is essential for tumorigenicity of basal breast cancer cells. Proc. Natl Acad. Sci. USA 116, 7353-7362 (2019). [PubMed: 30910979]

40. Pastushenko I et al. Identification of the tumour transition states occurring during EMT. Nature 556, 463-468 (2018). [PubMed: 29670281]

41. Gupta PB, Pastushenko I, Skibinski A, Blanpain C \& Kuperwasser C Phenotypic plasticity: driver of cancer initiation, progression, and therapy resistance. Cell Stem Cell 24, 65-78 (2019). [PubMed: 30554963]

42. Thompson EW \& Nagaraj SH Transition states that allow cancer to spread. Nature 556, 442-444 (2018) [PubMed: 29686367]

43. Hiscox $\mathrm{S}$ et al. Tamoxifen resistance in MCF7 cells promotes EMT-like behaviour and involves modulation of beta-catenin phosphorylation. Int. J. Cancer 118, 290-301 (2006). [PubMed: $16080193]$

44. Marin-Aguilera M et al. Epithelial-to-mesenchymal transition mediates docetaxel resistance and high risk of relapse in prostate cancer. Mol. Cancer Ther. 13, 1270-1284 (2014). [PubMed: 24659820]

45. Lesniak D et al. Spontaneous epithelial-mesenchymal transition and resistance to HER-2-targeted therapies in HER-2-positive luminal breast cancer. PLOS ONE 8, e71987 (2013). [PubMed: 23991019]

46. Miyazaki S et al. Anti-VEGF antibody therapy induces tumor hypoxia and stanniocalcin 2 expression and potentiates growth of human colon cancer xenografts. Int. J. Cancer 135, 295-307 (2014). [PubMed: 24375080]

47. Shintani $Y$ et al. Epithelial to mesenchymal transition is a determinant of sensitivity to chemoradiotherapy in non-small cell lung cancer. Ann. Thorac. Surg. 92, 1794-1804 (2011). [PubMed: 22051275]

48. Le Magnen C, Dutta A \& Abate-Shen C Optimizing mouse models for precision cancer prevention. Nat. Rev. Cancer 16, 187-196 (2016). [PubMed: 26893066]

49. Trimboli AJ et al. Direct evidence for epithelial-mesenchymal transitions in breast cancer. Cancer Res. 68, 937-945 (2008). [PubMed: 18245497]

50. Rios AC et al. Intraclonal plasticity in mammary tumors revealed through large-scale single-cell resolution 3D imaging. Cancer Cell 35, 953 (2019). [PubMed: 31185217]

51. Puram SV et al. Single-cell transcriptomic analysis of primary and metastatic tumor ecosystems in head and neck cancer. Cell 171, 1611-1624.e24 (2017). [PubMed: 29198524]

52. Redfern AD, et al. Predictive value of de novo and induced epithelial-mesenchymal transition in locally advanced breast cancer treated with neoadjuvant chemotherapy. Cancer Res. 76 10.1158/1538-7445.SABCS15-P1-05-0 (2016).

53. Jolly MK et al. Coupling the modules of EMT and sternness: a tunable 'sternness window' model. Oncotarget 6, 25161-25174 (2015). [PubMed: 26317796]

54. Nitta $\mathrm{T}$ et al. Prognostic significance of epithelial-mesenchymal transition-related markers in extrahepatic cholangiocarcinoma: comprehensive immunohistochemical study using a tissue microarray. Br. J. Cancer 111, 1363-1372 (2014). [PubMed: 25077440]

55. Sarrio D et al. Epithelial-mesenchymal transition in breast cancer relates to the basal-like phenotype. Cancer Res. 68, 989-997 (2008). [PubMed: 18281472]

56. Zhang $\mathrm{H}$ et al. Clinical significance of E-cadherin, ( $\beta$-catenin, vimentin and S100A4 expression in completely resected squamous cell lung carcinoma. J. Clin. Pathol. 66, 937-945 (2013). [PubMed: 23853314]

57. Wen $\mathrm{KC}$ et al. The role of EpCAM in tumor progression and the clinical prognosis of endometrial carcinoma. Gynecol. Oncol. 148, 383-392 (2018). [PubMed: 29208367]

58. Yamada $\mathrm{S}$ et al. Epithelial to mesenchymal transition is associated with shorter disease-free survival in hepatocellular carcinoma. Ann. Surg. Oncol. 21, 3882-3890 (2014). [PubMed: 24833103] 
59. Wang G, Pan J, Zhang L \& Wang C Overexpression of grainyhead-like transcription factor 2 is associated with poor prognosis in human pancreatic carcinoma. Oncol. Lett 17, 1491-1496 (2019). [PubMed: 30675204]

60 . Wu RS et al. OVOL2 antagonizes TGF- $\beta$ signaling to regulate epithelial to mesenchymal transition during mammary tumor metastasis. Oncotarget 8, 39401-39416 (2017). [PubMed: 28455959]

61. Shi M et al. MicroRNA-200 and microRNA-30 family as prognostic molecular signatures in ovarian cancer: a meta-analysis. Medicine 97, e11505 (2018). [PubMed: 30095616]

62. Powell E et al. A functional genomic screen in vivo identifies CEACAM5 as a clinically relevant driver of breast cancer metastasis. NPJ Breast Cancer 4, 9 (2018) [PubMed: 29736411]

63. Huang W, Martin EE, Burman B, Gonzalez ME \& Kleer CG The matricellular protein CCN6 (WISP3) decreases Notch1 and suppresses breast cancer initiating cells. Oncotarget 7, 2518025193 (2016) [PubMed: 26933820]

64. Prat A et al. Phenotypic and molecular characterization of the claudin-low intrinsic subtype of breast cancer. Breast Cancer Res. 12, R68 (2010). [PubMed: 20813035]

65. Tan TZ et al. Epithelial-mesenchymal transition spectrum quantification and its efficacy in deciphering survival and drug responses of cancer patients. EMBO Mol. Med. 6, 1279-1293 (2014). [PubMed: 25214461]

66. Blick T et al. Epithelial mesenchymal transition traits in human breast cancer cell lines parallel the CD44hi/CD24lo/-stem cell phenotype in human breast cancer. J. Mammary Gland. Biol. Neoplasia 15, 235-252 (2010). [PubMed: 20521089]

67. Kotiyal S \& Bhattacharya S Breast cancer stem cells, EMT and therapeutic targets. Biochem. Biophys. Res. Commun. 453, 112-166 (2014). [PubMed: 25261721]

68. Mani SA et al. The epithelial-mesenchymal transition generates cells with properties of stem cells. Cell 133, 704-715 (2008). [PubMed: 18485877]

69. Morel AP et al. Generation of breast cancer stem cells through epithelial-mesenchymal transition. PLOS ONE 3, e2888 (2008). [PubMed: 18682804]

70. Shibue T \& Weinberg RA EMT, CSCs, and drug resistance: the mechanistic link and clinical implications. Nat. Rev. Clin. Oncol. 14, 611-629 (2017) [PubMed: 28397828]

71. Ocaña $\mathrm{OH}$ et al. Metastatic colonization requires the repression of the epithelial-mesenchymal transition inducer Prrx1. Cancer Cell 22, 709-724 (2012). [PubMed: 23201163]

72. Zhu QC, Gao RY, Wu W \& Qin HL Epithelial-mesenchymal transition and its role in the pathogenesis of colorectal cancer. Asian Pac. J. Cancer Prev. 14, 2689-2698 (2013). [PubMed: 23803016]

73. Xu S et al. Characterization of mouse models of early pancreatic lesions induced by alcohol and chronic pancreatitis. Pancreas 44, 882-887 (2015) [PubMed: 26166469]

74. Lee J et al. Human primary epithelial cells acquire an epithelial-mesenchymal-transition phenotype during long-term infection by the oral opportunistic pathogen, Porphyromonas gingivalis. Front. Cell Infect. Microbiol. 7, 493 (2017). [PubMed: 29250491]

75. Yang SZ et al. HBx protein induces EMT through c-Src activation in SMMC-7721 hepatoma cell line. Biochem. Biophys. Res. Commun. 382, 555-560 (2009) [PubMed: 19302982]

76. Shen HJ et al. Cigarette smoke-induced alveolar epithelial-mesenchymal transition is mediated by Rac1 activation. Biochim. Biophys. Acta 1840, 1838-1849 (2014) [PubMed: 24508121]

77. Wang Y, Xu M, Ke Z \& Luo J Cellular and molecular mechanism underlying alcohol-induced aggressiveness of breast cancer. Pharmacol. Res. 115, 299-308 (2017). [PubMed: 27939360]

78. Puisieux A, Brabletz T \& Caramel J Oncogenic roles of EMT-inducing transcription factors. Nat. Cell Biol. 16, 488-494 (2014). [PubMed: 24875735]

79. Li J et al. The EMT transcription factor Zeb2 controls adult murine hematopoietic differentiation by regulating cytokine signaling. Blood 129, 460-472 (2017). [PubMed: 27683414]

80. Liskova $\mathrm{P}$ et al. Ectopic GRHL2 expression due to noncoding mutations promotes cell state transition and causes posterior polymorphous corneal dystrophy 4. Am. J. Hum. Genet. 102, 447459 (2018). [PubMed: 29499165]

81. Sharma VP et al. Mutations in TCF12, encoding a basic helix-loop-helix partner of TWIST1, are a frequent cause of coronal craniosynostosis. Nat. Genet. 45, 304-307 (2013). [PubMed: 23354436] 
82. Hugo HJ et al. Epithelial requirement for in vitro proliferation and xenograft growth and metastasis of MDA-MB-468 human breast cancer cells: oncogenic rather than tumor-suppressive role of Ecadherin. Breast Cancer Res. 19, 86 (2017). [PubMed: 28750639]

83. Gunasinghe NP, Wells A, Thompson EW \& Hugo HJ Mesenchymal-epithelial transition (MET) as a mechanism for metastatic colonisation in breast cancer. Cancer Metastasis Rev. 31,469-478 (2012). [PubMed: 22729277]

84. Joseph JP, Harishankar MK, Pillai AA \& Devi A Hypoxia induced EMT: a review on the mechanism of tumor progression and metastasis in OSCC. Oral. Oncol. 80, 23-32 (2018). [PubMed: 29706185]

85. Wei $\mathrm{L}$ et al. Leptin promotes epithelial-mesenchymal transition of breast cancer via the upregulation of pyruvate kinase M2. J. Exp. Clin. Cancer Res. 35, 166 (2016). [PubMed: 27769315]

86. Feng $\mathrm{H}$ et al. Leptin promotes metastasis by inducing an epithelial-mesenchymal transition in A549 lung cancer cells. Oncol. Res. 21, 165-171 (2013). [PubMed: 24512731]

87. Peppicelli S, Bianchini F, Torre E \& Calorini L Contribution of acidic melanoma cells undergoing epithelial-to-mesenchymal transition to aggressiveness of non-acidic melanoma cells. Clin. Exp. Metastasis 31, 423-433 (2014). [PubMed: 24469963]

88. Deng S et al. MiR-652 inhibits acidic microenvironment-induced epithelial-mesenchymal transition of pancreatic cancer cells by targeting ZEB1. Oncotarget 6, 39661-39675 (2015). [PubMed: 26498682]

89. Suzuki A, Maeda T, Baba Y, Shimamura K \& Kato Y Acidic extracellular pH promotes epithelial mesenchymal transition in Lewis lung carcinoma model. Cancer Cell Int. 14, 129 (2014). [PubMed: 25493076]

90. Marin-Hernandez A et al. Hypoglycemia enhances epithelial-mesenchymal transition and invasiveness, and restrains the Warburg phenotype, in hypoxic HeLa cell cultures and microspheroids. J. Cell Physiol. 232, 1346-1359 (2017). [PubMed: 27661776]

91. Wei SC et al. Matrix stiffness drives epithelial-mesenchymal transition and tumour metastasis through a TWIST1-G3BP2 mechanotransduction pathway. Nat. Cell Biol. 17, 678-688 (2015). [PubMed: 25893917]

92. Rice AJ et al. Matrix stiffness induces epithelial-mesenchymal transition and promotes chemoresistance in pancreatic cancer cells. Oncogenesis 6, e352 (2017). [PubMed: 28671675]

93. Brabletz $\mathrm{T}$ et al. Nuclear overexpression of the oncoprotein $\beta$-catenin in colorectal cancer is localized predominantly at the invasion front. Pathol. Res. Pract. 194, 701-704 (1998). [PubMed: 9820866]

94. Brabletz $\mathrm{T}$ et al. Variable $\beta$-catenin expression in colorectal cancers indicates tumor progression driven by the tumor environment. Proc. Natl Acad. Sci. USA 98, 10356-10361 (2001). [PubMed: $11526241]$

95. Zlobec I \& Lugli A Epithelial mesenchymal transition and tumor budding in aggressive colorectal cancer: tumor budding as oncotarget. Oncotarget 1, 651-661 (2010). [PubMed: 21317460]

96. Bronsert $\mathrm{P}$ et al. Cancer cell invasion and EMT marker expression: a three-dimensional study of the human cancer-host interface. J. Pathol. 234, 410-422 (2014). [PubMed: 25081610]

97. Zhao $\mathrm{Z}$ et al. In vivo visualization and characterization of epithelial-mesenchymal transition in breast tumors. Cancer Res. 76, 2094-2104 (2016). [PubMed: 26893478]

98. Del Pozo Martin Y et al. Mesenchymal cancer cell-stroma crosstalk promotes niche activation, epithelial reversion, and metastatic colonization. Cell Rep. 13, 2456-2469 (2015). [PubMed: 26670048]

99. Puram SV, Parikh AS \& Tirosh I Single cell RNA-seq highlights a role for a partial EMT in head and neck cancer. Mol. Cell Oncol. 5, e1448244 (2018). [PubMed: 30250901]

100. Yu M et al. Circulating breast tumor cells exhibit dynamic changes in epithelial and mesenchymal composition. Science 339, 580-584 (2013). [PubMed: 23372014]

101. Zheng $X$ et al. Epithelial-to-mesenchymal transition is dispensable for metastasis but induces chemoresistance in pancreatic cancer. Nature 527, 525-530 (2015). [PubMed: 26560028] 
102. Huang Z, Yang M, Li Y, Yang F \& Feng Y Exosomes derived from hypoxic colorectal cancer cells transfer Wnt4 to normoxic cells to elicit a prometastatic phenotype. Int. J. Biol. Sci 14, 2094-2102 (2018). [PubMed: 30585272]

103. El-Sayed IY et al. Extracellular vesicles released by mesenchymal-like prostate carcinoma cells modulate EMT state of recipient epithelial-like carcinoma cells through regulation of AR signaling. Cancer Lett. 410, 100-111 (2017). [PubMed: 28935391]

104. Lobb RJ et al. Exosomes derived from mesenchymal non-small cell lung cancer cells promote chemoresistance. Int. J. Cancer 141, 614-620 (2017). [PubMed: 28445609]

105. Wu S et al. Classification of circulating tumor cells by epithelial-mesenchymal transition markers. PLOS ONE 10, e0123976 (2015). [PubMed: 25909322]

106. Markiewicz A et al. Mesenchymal phenotype of CTC-enriched blood fraction and lymph node metastasis formation potential. PLOS ONE 9, e93901 (2014). [PubMed: 24709997]

107. Strati A et al. Gene expression profile of circulating tumor cells in breast cancer by RT-qPCR. BMC Cancer 11, 422 (2011). [PubMed: 21967632]

108. Sarioglu AF et al. A microfluidic device for label-free, physical capture of circulating tumor cell clusters. Nat. Methods 12, 685-691 (2015). [PubMed: 25984697]

109. Aceto $\mathrm{N}$ et al. Circulating tumor cell clusters are oligoclonal precursors of breast cancer metastasis. Cell 158, 1110-1122 (2014). [PubMed: 25171411]

110. Ting DT et al. Single-cell RNA sequencing identifies extracellular matrix gene expression by pancreatic circulating tumor cells. Cell Rep. 8, 1905-1918 (2014). [PubMed: 25242334]

111. Gkountela $\mathrm{S}$ et al. Circulating tumor cell clustering shapes DNA methylation to enable metastasis seeding. Cell 176, 98-112.e14 (2019). [PubMed: 30633912]

112. Papadaki MA et al. Circulating tumor cells with stemness and epithelial-to-mesenchymal transition features are chemoresistant and predictive of poor outcome in metastatic breast cancer. Mol. Cancer Ther. 18, 437-447 (2019). [PubMed: 30401696]

113. de Wit $\mathrm{S}$ et al. EpCAMhlgh and EpCAMlow circulating tumor cells in metastatic prostate and breast cancer patients. Oncotarget 9, 35705-35716 (2018). [PubMed: 30479699]

114. Driemel C et al. Context-dependent adaption of EpCAM expression in early systemic esophageal cancer. Oncogene 33, 4904-4915 (2014). [PubMed: 24141784]

115. Liu C et al. Epithelial-type systemic breast carcinoma cells with a restricted mesenchymal transition are a major source of metastasis. Sci. Adv 5, eaav4275 (2019). [PubMed: 31223646]

116. Hiraga T Hypoxic microenvironment and metastatic bone disease. Int. J. Mol. Sci. 19, E3523 (2018). [PubMed: 30423905]

117. Chao Y, Wu Q, Acquafondata M, Dhir R \& Wells A Partial mesenchymal to epithelial reverting transition in breast and prostate cancer metastases. Cancer Microenviron. 5, 19-28 (2012). [PubMed: 21892699]

118. Chao Y, Wu Q, Shepard C \& Wells A Hepatocyte induced re-expression of E-cadherin in breast and prostate cancer cells increases chemoresistance. Clin. Exp. Metastasis 29, 39-50 (2012). [PubMed: 21964676]

119. Chao YL, Shepard CR \& Wells A Breast carcinoma cells re-express E-cadherin during mesenchymal to epithelial reverting transition. Mol. Cancer 9, 179 (2010). [PubMed: 20609236]

120. Kowalski P J., Rubin, M. A. \& Kleer, C. G. E-cadherin expression in primary carcinomas of the breast and its distant metastases. Breast Cancer Res. 5, R217-222 (2003). [PubMed: 14580257]

121. Wells A, Chao YL, Grahovac J, Wu Q \& Lauffenburger DA Epithelial and mesenchymal phenotypic switchings modulate cell motility in metastasis. Front. Biosci. 16, 815-837 (2011).

122. Saha B et al. Overexpression of E-cadherin protein in metastatic breast cancer cells in bone. Anticancer. Res. 27, 3903-3908 (2007). [PubMed: 18225549]

123. Bhullar DS et al. Biomarker concordance between primary colorectal cancer and its metastases. EBioMedicine 40, 363-374 (2019). [PubMed: 30733075]

124. Choi $\mathrm{W}$ et al. p63 expression defines a lethal subset of muscle-invasive bladder cancers. PLOS ONE 7, e30206 (2012). [PubMed: 22253920] 
125. Chaffer CL et al. Mesenchymal-to-epithelial transition facilitates bladder cancer metastasis: role of fibroblast growth factor receptor-2. Cancer Res. 66, 11271-11278 (2006). [PubMed: 17145872]

126. Celia-Terrassa $\mathrm{T}$ et al. Epithelial-mesenchymal transition can suppress major attributes of human epithelial tumor-initiating cells. J. Clin. Invest. 122, 1849-1868 (2012). [PubMed: 22505459]

127. Dykxhoorn DM et al. miR-200 enhances mouse breast cancer cell colonization to form distant metastases. PLOS ONE 4, e7181 (2009). [PubMed: 19787069]

128. Shamir ER et al. Twist1-induced dissemination preserves epithelial identity and requires Ecadherin. J. Cell Biol. 204, 839-856 (2014). [PubMed: 24590176]

129. Shamir ER, Coutinho K, Georgess D, Auer M \& Ewald AJ Twist1-positive epithelial cells retain adhesive and proliferative capacity throughout dissemination. Biol. Open 5, 1216-1228 (2016). [PubMed: 27402962]

130. Korpal M et al. Direct targeting of Sec23a by miR-200s influences cancer cell secretome and promotes metastatic colonization. Nat. Med. 17, 1101-1108 (2011). [PubMed: 21822286]

131. Celia-Terrassa $\mathrm{T}$ et al. Hysteresis control of epithelial-mesenchymal transition dynamics conveys a distinct program with enhanced metastatic ability. Nat. Commun. 9, 5005 (2018). [PubMed: 30479345]

132. Tran HD et al. Transient SNAIL1 expression is necessary for metastatic competence in breast cancer. Cancer Res. 74, 6330-6340 (2014). [PubMed: 25164016]

133. Tsai JH, Donaher JL, Murphy DA, Chau S \& Yang J Spatiotemporal regulation of epithelialmesenchymal transition is essential for squamous cell carcinoma metastasis. Cancer Cell 22, 725-736 (2012). [PubMed: 23201165]

134. Stankic $\mathrm{M}$ et al. TGF $\beta$-Id1 signaling opposes Twist1 and promotes metastatic colonization via a mesenchymal-to-epithelial transition. Cell Rep. 5, 1228-1242 (2013). [PubMed: 24332369]

135. Behnsawy HM, Miyake H, Harada K \& Fujisawa M Expression patterns of epithelialmesenchymal transition markers in localized prostate cancer: significance in clinicopathological outcomes following radical prostatectomy. BJU Int. 111,30-37 (2013). [PubMed: 23107154]

136. Gravdal K, Halvorsen OJ, Haukaas SA \& Akslen LA A switch from E-cadherin to N-cadherin expression indicates epithelial to mesenchymal transition and is of strong and independent importance for the progress of prostate cancer. Clin. Cancer Res. 13, 7003-7011 (2007). [PubMed: 18056176]

137. Sethi S, Macoska J, Chen W \& Sarkar FH Molecular signature of epithelial-mesenchymal transition (EMT) in human prostate cancer bone metastasis. Am. J. Trans!. Res 3, 90-99 (2010).

138. Yamamoto $\mathrm{M}$ et al. Intratumoral bidirectional transitions between epithelial and mesenchymal cells in triple-negative breast cancer. Cancer Sci. 108, 1210-1222 (2017). [PubMed: 28371195]

139. Gao D et al. Myeloid progenitor cells in the premetastatic lung promote metastases by inducing mesenchymal to epithelial transition. Cancer Res. 72, 1384-1394 (2012). [PubMed: 22282653]

140. Yates CC, Shepard CR, Stolz DB \& Wells A Co-culturing human prostate carcinoma cells with hepatocytes leads to increased expression of E-cadherin. Br. J. Cancer 96, 1246-1252 (2007). [PubMed: 17406365]

141. Aiello NM et al. Metastatic progression is associated with dynamic changes in the local microenvironment. Nat. Commun. 7, 12819 (2016). [PubMed: 27628423]

142. Ackland ML et al. Epidermal growth factor-induced epithelio-mesenchymal transition in human breast carcinoma cells. Lab. Invest. 83, 435-448 (2003). [PubMed: 12649344]

143. Dumont $\mathrm{N}$ et al. Sustained induction of epithelial to mesenchymal transition activates DNA methylation of genes silenced in basal-like breast cancers. Proc. Natl Acad. Sci. USA 105, 14867-14872 (2008). [PubMed: 18806226]

144. Ye X et al. Upholding a role for EMT in breast cancer metastasis. Nature 547, E1-E3 (2017). [PubMed: 28682326]

145. Aiello NM et al. Upholding a role for EMT in pancreatic cancer metastasis. Nature 547, E7-E8 (2017). [PubMed: 28682339]

146. Xu Y et al. Breast tumor cell-specific knockout of Twist 1 inhibits cancer cell plasticity, dissemination, and lung metastasis in mice. Proc. Natl Acad. Sci. USA 114, 11494-11499 (2017). [PubMed: 29073077] 
147. Fischer KR, Altorki NK, Mittal V \& Gao D. Fischer et al. reply. Nature 547, E5-E6 (2017). [PubMed: 28682327]

148. Somarelli JA et al. Distinct routes to metastasis: plasticity-dependent and plasticity-independent pathways. Oncogene 35, 4302-4311 (2016). [PubMed: 26751776]

149. Rhim AD et al. EMT and dissemination precede pancreatic tumor formation. Cell 148, 349-361 (2012). [PubMed: 22265420]

150. Castano $\mathrm{Z}$ et al. IL-1 $\beta$ inflammatory response driven by primary breast cancer prevents metastasis-initiating cell colonization. Nat. Cell Biol. 20, 1084-1097 (2018). [PubMed: 30154549]

151. Cheng C, Qin Y, Li Y, Pan J \& Wang J Expression of Twist protein in colorectal carcinoma and its effect on proliferation and invasion of colorectal cancer cells. Pak. J. Pharm. Sci. 30, 641-645 (2017). [PubMed: 28650334]

152. Qi J et al. SNAI1 promotes the development of HCC through the enhancement of proliferation and inhibition of apoptosis. FEBS Open Bio. 6, 326-337 (2016).

153. Title AC et al. Genetic dissection of the miR-200-Zeb1 axis reveals its importance in tumor differentiation and invasion. Nat. Commun. 9, 4671 (2018). [PubMed: 30405106]

154. Yang $\mathrm{C}$ et al. Long noncoding RNA HCP5 contributes to epithelial-mesenchymal transition in colorectal cancer through ZEB1 activation and interacting with miR-139-5p. Am J. Transl. Res 11, 953-963 (2019). [PubMed: 30899394]

155. Mejlvang J et al. Direct repression of cyclin D1 by SIP1 attenuates cell cycle progression in cells undergoing an epithelial mesenchymal transition. Mol. Biol. Cell 18, 4615-4624 (2007). [PubMed: 17855508]

156. George JT, Jolly MK, Xu S, Somarelli JA \& Levine H Survival outcomes in cancer patients predicted by a partial EMT gene expression scoring metric. Cancer Res. 77, 6415-6428 (2017). [PubMed: 28947416]

157. Foroutan $\mathrm{M}$ et al. Single sample scoring of molecular phenotypes. BMC Bioinformatics 19, 404 (2018). [PubMed: 30400809]

158. Goossens N, Hoshida Y \& Aguirre-Ghiso JA Origin and interpretation of cancer transcriptome profiling: the essential role of the stroma in determining prognosis and drug resistance. EMBO Mol. Med. 7, 1385-1387 (2015). [PubMed: 26240076]

159. Dunne PD et al. Challenging the cancer molecular stratification dogma: intratumoral heterogeneity undermines consensus molecular subtypes and potential diagnostic value in colorectal cancer. Clin. Cancer Res. 22, 4095-4104 (2016). [PubMed: 27151745]

160. Lawson DA et al. Single-cell analysis reveals a stemcell program in human metastatic breast cancer cells. Nature 526, 131-135 (2015). [PubMed: 26416748]

161. Cook DP \& Vanderhyden BC Comparing transcriptional dynamics of the epithelial-mesenchymal transition. Preprint at bioRxiv 10.1101/732412 (2019).

162. Bhatia $S$ et al. Interrogation of phenotypic plasticity between epithelial and mesenchymal states in breast cancer. J. Clin. Med. 8, E893 (2019). [PubMed: 31234417]

163. Jolly MK et al. Stability of the hybrid epithelial/ mesenchymal phenotype. Oncotarget 7, 2706727084 (2016). [PubMed: 27008704]

164. Chaffer CL et al. Poised chromatin at the ZEB1 promoter enables breast cancer cell plasticity and enhances tumorigenicity. Cell 154, 61-74 (2013). [PubMed: 23827675]

165. Redfern AD, Spalding LJ \& Thompson EW The Kraken Wakes: induced EMT as a driver of tumour aggression and poor outcome. Clin. Exp. Metastasis 35, 285-308 (2018). [PubMed: 29948647]

166. Puhr M et al. Epithelial-to-mesenchymal transition leads to docetaxel resistance in prostate cancer and is mediated by reduced expression of miR-200c and miR-205. Am. J. Pathol. 181, 21882201 (2012). [PubMed: 23041061]

167. Creighton CJ et al. Residual breast cancers after conventional therapy display mesenchymal as well as tumor-initiating features. Proc. Natl Acad. Sci. USA 106, 13820-13825 (2009). [PubMed: 19666588] 
168. Qu H, Fang L, Duan L \& Long X [Expression of ABCG2 and p-glycoprotein in residual breast cancer tissue after chemotherapy and their correlation with epithelial-mesenchymal transition]. Zhonghua Bing Li Xue Za Zhi 43, 236-240 (2014). [PubMed: 24915813]

169. Hara $\mathrm{J}$ et al. Mesenchymal phenotype after chemotherapy is associated with chemoresistance and poor clinical outcome in esophageal cancer. Oncol. Rep. 31, 589-596 (2014). [PubMed: 24297447]

170. Bhangu A et al. The role of epithelial mesenchymal transition and resistance to neoadjuvant therapy in locally advanced rectal cancer. Colorectal Dis. 16, O 133-143 (2014).

171. Kawamoto A et al. Radiation induces epithelial-mesenchymal transition in colorectal cancer cells. Oncol. Rep. 27, 51-57 (2012). [PubMed: 21971767]

172. Martin OA, Anderson RL, Narayan K \& MacManus MP Does the mobilization of circulating tumour cells during cancer therapy cause metastasis? Nat. Rev. Clin. Oncol 14, 32-44 (2017). [PubMed: 27550857]

173. Martin OA et al. Mobilization of viable tumor cells into the circulation during radiation therapy. Int. J. Radiat. Oncol. Biol. Phys. 88, 395-403 (2014). [PubMed: 24315565]

174. Alves CL, Elias D, Lyng MB, Bak M \& Ditzel HJ SNAI2 upregulation is associated with an aggressive phenotype in fulvestrant-resistant breast cancer cells and is an indicator of poor response to endocrine therapy in estrogen receptor-positive metastatic breast cancer. Breast Cancer Res. 20, 60 (2018). [PubMed: 29921289]

175. Sun $Y$ et al. Androgen deprivation causes epithelial-mesenchymal transition in the prostate: implications for androgen-deprivation therapy. Cancer Res. 72, 527-536 (2012). [PubMed: 22108827]

176. Frederick BA et al. Epithelial to mesenchymal transition predicts gefitinib resistance in cell lines of head and neck squamous cell carcinoma and non-small cell lung carcinoma. Mol. Cancer Ther. 6, 1683-1691 (2007). [PubMed: 17541031]

177. Kim HR et al. Epithelial-mesenchymal transition leads to crizotinib resistance in H2228 lung cancer cells with EML4-ALK translocation. Mol. Oncol 7, 1093-1102 (2013). [PubMed: 23993685]

178. Huang D et al. Hypoxia induces actin cytoskeleton remodeling by regulating the binding of CAPZA1 to F-actin via PIP2 to drive EMT in hepatocellular carcinoma. Cancer Lett. 448, $117-$ 127 (2019). [PubMed: 30742939]

179. Singh SK, Mishra MK \& Singh R Hypoxia-inducible factor-1a induces CX3CR1 expression and promotes the epithelial to mesenchymal transition (EMT) in ovarian cancer cells. J. Ovarian. Res. 12, 42 (2019). [PubMed: 31077234]

180. Yang Z, Yu W, Huang R, Ye M \& Min Z SIRT6/HIF-1a axis promotes papillary thyroid cancer progression by inducing epithelial-mesenchymal transition. Cancer CellInt. 19, 17 (2019).

181. Zhang J et al. Hypoxia-induced LncRNA PCGEM1 promotes invasion and metastasis of gastric cancer through regulating SNAI1. Clin. Transl Oncol 21, 1142-1151 (2019). [PubMed: 30690667]

182. Sauvant $\mathrm{C}$ et al. Acidosis induces multi-drug resistance in rat prostate cancer cells (AT1) in vitro and in vivo by increasing the activity of the p-glycoprotein via activation of p38. Int. J. Cancer 123, 2532-2542 (2008). [PubMed: 18729196]

183. Thews O \& Riemann A Tumor $\mathrm{pH}$ and metastasis: a malignant process beyond hypoxia. Cancer Metastasis Rev. 38, 113-129 (2019). [PubMed: 30607627]

184. Chockley PJ \& Keshamouni VG Immunological consequences of epithelial-mesenchymal transition in tumor progression. J. Immunol. 197, 691-698 (2016). [PubMed: 27431984]

185. Terry $\mathrm{S}$ et al. New insights into the role of EMT in tumor immune escape. Mol. Oncol. 11, 824846 (2017). [PubMed: 28614624]

186. Davis FM, Stewart TA, Thompson EW \& Monteith GR Targeting EMT in cancer: opportunities for pharmacological intervention. Trends Pharmacol. Sci. 35, 479-488 (2014). [PubMed: 25042456]

187. Behbahani $\mathrm{G}$ et al. MicroRNA-mediated post-transcriptional regulation of epithelial to mesenchymal transition in cancer. Pathol. Oncol. Res. 23, 1-12 (2017). [PubMed: 27590333] 
188. Li Y et al. Up-regulation of $m i R-200$ and let-7 by natural agents leads to the reversal of epithelialto-mesenchymal transition in gemcitabine-resistant pancreatic cancer cells. Cancer Res. 69, 6704-6712 (2009). [PubMed: 19654291]

189. Meidhof S et al. ZEB1-associated drug resistance in cancer cells is reversed by the class I HDAC inhibitor mocetinostat. EMBO Mol. Med. 7, 831-847 (2015). [PubMed: 25872941]

190. Beg MS et al. Phase I study of MRX34, a liposomal miR-34a mimic, administered twice weekly in patients with advanced solid tumors. Invest. New Drugs 35, 180-188 (2017). [PubMed: 27917453]

191. van Zandwijk N et al. Safety and activity of microRNA-loaded minicells in patients with recurrent malignant pleural mesothelioma: a first-in-man, phase 1, open-label, dose-escalation study. Lancet Oncol. 18, 1386-1396 (2017). [PubMed: 28870611]

192. Scherbakov AM, Andreeva OE, Shatskaya VA \& Krasil'nikov MA The relationships between snail1 and estrogen receptor signaling in breast cancer cells. J. CellBiochem. 113, 2147-2155 (2012).

193. Haslehurst AM et al. EMT transcription factors snail and slug directly contribute to cisplatin resistance in ovarian cancer. BMC Cancer 12, 91 (2012). [PubMed: 22429801]

194. Witta SE et al. Restoring E-cadherin expression increases sensitivity to epidermal growth factor receptor inhibitors in lung cancer cell lines. Cancer Res. 66, 944-950 (2006). [PubMed: 16424029]

195. Baylis F \& McLeod M First-in-human phase 1 CRISPR gene editing cancer trials: are we ready? Curr. Gene Ther. 17, 309-319 (2017). [PubMed: 29173170]

196. Garcia Bloj B et al. Waking up dormant tumor suppressor genes with zinc fingers, TALEs and the CRISPR/dCas9 system. Oncotarget 7, 60535-60554 (2016). [PubMed: 27528034]

197. Moses C et al. Activating PTEN tumor suppressor expression with the CRISPR/dCas9 system. Mol. Ther. Nucleic Acids 14, 287-300 (2019). [PubMed: 30654190]

198. Huber MA, Kraut N \& Beug H Molecular requirements for epithelial-mesenchymal transition during tumor progression. Curr. Opin. Cell Biol. 17, 548-558 (2005). [PubMed: 16098727]

199. Said NA \& Williams ED Growth factors in induction of epithelial-mesenchymal transition and metastasis. Cells Tissues Organs 193, 85-97 (2011). [PubMed: 21051862]

200. Loh YN et al. The Wnt signalling pathway is upregulated in an in vitro model of acquired tamoxifen resistant breast cancer. BMC Cancer 13, 174 (2013). [PubMed: 23547709]

201. Buonato JM \& Lazzara MJ ERK1/2 blockade prevents epithelial-mesenchymal transition in lung cancer cells and promotes their sensitivity to EGFR inhibition. Cancer Res. 74, 309-319 (2014). [PubMed: 24108744]

202. Liu J et al. Association of tumour-associated macrophages with cancer cell EMT, invasion, and metastasis of Kazakh oesophageal squamous cell cancer. Diagn. Pathol 14, 55 (2019). [PubMed: 31186031]

203. Zhang Q et al. Interaction of transforming growth factor- $\beta$-Smads/microRNA-362-3p/CD82 mediated by M2 macrophages promotes the process of epithelial-mesenchymal transition in hepatocellular carcinoma cells. Cancer Sci. 110, 2507-2519 (2019). [PubMed: 31215741]

204. Li S et al. Tumor-associated neutrophils induce EMT by IL-17a to promote migration and invasion in gastric cancer cells. J. Exp. Clin. Cancer Res 38, 6 (2019). [PubMed: 30616627]

205. Donnarumma E et al. Cancer-associated fibroblasts release exosomal microRNAs that dictate an aggressive phenotype in breast cancer. Oncotarget 8, 19592-19608 (2017). [PubMed: 28121625]

206. Raviraj V et al. Dormant but migratory tumour cells in desmoplastic stroma of invasive ductal carcinomas. Clin. Exp. Metastasis 29, 273-292 (2012). [PubMed: 22271313]

207. Lee $\mathrm{J}$ et al. The metastasis suppressor CD82/KAI1 inhibits fibronectin adhesion-induced epithelial-to-mesenchymal transition in prostate cancer cells by repressing the associated integrin signaling. Oncotarget 8, 1641-1654 (2017). [PubMed: 27926483]

208. Jin $\mathrm{H}$ et al. Targeting lipid metabolism to overcome EMT-associated drug resistance via integrin ( $\beta 3$ /FAK pathway and tumor-associated macrophage repolarization using legumain-activatable delivery. Theranostics 9, 265-278 (2019). [PubMed: 30662566]

209. Fang J et al. A potent immunotoxin targeting fibroblast activation protein for treatment of breast cancer in mice. Int. J. Cancer 138, 1013-1023 (2016). [PubMed: 26334777] 
210. Loeffler I, Liebisch M \& Wolf G Collagen VIII influences epithelial phenotypic changes in experimental diabetic nephropathy. Am. J. Physiol. Ren. Physiol 303, F733-745 (2012).

211. Terashima $\mathrm{M}$ et al. Synergistic antitumor effects of S-1 with eribulin in vitro and in vivo for triplenegative breast cancer cell lines. Springerplus 3, 417 (2014). [PubMed: 25140293]

212. Chiu LY et al. The ERK-ZEB1 pathway mediates epithelial-mesenchymal transition in pemetrexed resistant lung cancer cells with suppression by vinca alkaloids. Oncogene 36, 242 253 (2017). [PubMed: 27270426]

213. Aparicio LA et al. Role of the microtubule-targeting drug vinflunine on cell-cell adhesions in bladder epithelial tumour cells. BMC Cancer 14, 507 (2014). [PubMed: 25012153]

214. Demetri G et al. Activity of eribulin in patients with advanced liposarcoma demonstrated in a subgroup analysis from a randomized phase III study of eribulin versus dacarbazine. J. Clin. Oncol. 35, 3433-3439 (2017). [PubMed: 28854066]

215. Twelves $\mathrm{C}$ et al. Efficacy of eribulin in women with metastatic breast cancer: a pooled analysis of two phase 3 studies. Breast Cancer Res. Treat. 148, 553-561 (2014). [PubMed: 25381136]

216. Ishay-Ronen D et al. Gain fat-lose metastasis: converting invasive breast cancer cells into adipocytes inhibits cancer metastasis. Cancer Cell 35, 17-32.e6 (2019). [PubMed: 30645973]

217. Gupta $P$ et al. Identification of selective inhibitors of cancer stem cells by high-throughput screening. Cell 138, 645-659 (2009). [PubMed: 19682730]

218. Sánchez Tilló E et al. The EMT activator ZEB1 promotes tumor growth and determines differential response to chemotherapy in mantle cell lymphoma. Cell Death Differ. 21, 247-257 (2014). [PubMed: 24013721]

219. Qu C et al. Metformin reverses multidrug resistance and epithelial-mesenchymal transition (EMT) via activating AMP-activated protein kinase (AMPK) in human breast cancer cells. Mol. Cell Biochem. 386, 63-71 (2014). [PubMed: 24096736]

220. Jia D et al. OVOL guides the epithelial-hybrid-mesenchymal transition. Oncotarget 6, 1543615448 (2015). [PubMed: 25944618]

221. Cursons J et al. Stimulus-dependent differences in signalling regulate epithelial-mesenchymal plasticity and change the effects of drugs in breast cancer cell lines. Cell Commun Signal 13, 26 (2015). [PubMed: 25975820]

222. Twelves C et al. "New" metastases are associated with a poorer prognosis than growth of preexisting metastases in patients with metastatic breast cancer treated with chemotherapy. Breast Cancer Res. 17, 150 (2015). [PubMed: 27391598]

223. López-Novoa JM \& Nieto MA Inflammation and EMT: an alliance towards organ fibrosis and cancer progression. EMBO Mol. Med. 1,303-314 (2009). [PubMed: 20049734]

224. Latil M et al. Cell-type-specific chromatin states differentially prime squamous cell carcinoma tumor-initiating cells for epithelial to mesenchymal transition. Cell Stem Cell 20, 191-204.e5 (2017). [PubMed: 27889319]

225. Chen Y et al. Dual reporter genetic mouse models of pancreatic cancer identify an epithelial-tomesenchymal transition-independent metastasis program. EMBO Mol. Med. 10 10.15252/ emmm.201809085 (2018).

226. Ye X et al. Distinct EMT programs control normal mammary stem cells and tumour-initiating cells. Nature 525, 256-260 (2015). [PubMed: 26331542]

227. Aiello NM et al. EMT subtype influences epithelial plasticity and mode of cell migration. Dev. Cell 45, 681-695.e4 (2018). [PubMed: 29920274]

228. Tsuji $\mathrm{T}$ et al. Epithelial-mesenchymal transition induced by growth suppressor p12CDK2-AP1 promotes tumor cell local invasion but suppresses distant colony growth. Cancer Res. 68, 1037710386 (2008). [PubMed: 19074907]

229. Neelakantan D et al. EMT cells increase breast cancer metastasis via paracrine GLI activation in neighbouring tumour cells. Nat. Commun. 8, 15773 (2017). [PubMed: 28604738]

230. Neelakantan D, Drasin DJ \& Ford HL Intratumoral heterogeneity: clonal cooperation in epithelial-to-mesenchymal transition and metastasis. CellAdh. Migr. 9, 265-276 (2015).

231. Zhou H, Neelakantan D \& Ford HL Clonal cooperativity in heterogenous cancers. Semin. Cell Dev. Biol. 64, 79-89 (2017). [PubMed: 27582427] 
232. Cheung KJ et al. Polyclonal breast cancer metastases arise from collective dissemination of keratin 14-expressing tumor cell clusters. Proc. Natl Acad. Sci. USA 113, E854-863 (2016). [PubMed: 26831077]

233. Maddipati R \& Stanger BZ Pancreatic cancer metastases harbor evidence of polyclonality. CancerDiscov. 5, 1086-1097 (2015).

234. Liu L, Ye Y \& Zhu X MMP-9 secreted by tumor associated macrophages promoted gastric cancer metastasis through a PI3K/AKT/Snail pathway. Biomed. Pharmacother. 117, 109096 (2019). [PubMed: 31202170]

235. Zou J et al. Secreted TGF-p-induced protein promotes aggressive progression in bladder cancer cells. Cancer Manag. Res. 11,6995-7006 (2019). [PubMed: 31440088]

236. Hirai $\mathrm{M}$ et al. Regulation of PD-L1 expression in a high-grade invasive human oral squamous cell carcinoma microenvironment. Int. J. Oncol. 50, 41-48 (2017). [PubMed: 27922697]

237. Yao J et al. Altered expression and splicing of ESRP1 in malignant melanoma correlates with epithelial-mesenchymal status and tumor-associated immune cytolytic activity. Cancer Immunol. Res. 4, 552-561 (2016). [PubMed: 27045022]

238. Kudo-Saito C, Shirako H, Takeuchi T \& Kawakami Y Cancer metastasis is accelerated through immunosuppression during Snail-induced EMT of cancer cells. Cancer Cell 15, 195-206 (2009). [PubMed: 19249678]

239. Chen L et al. Metastasis is regulated via microRNA-200/ZEB1 axis control of tumour cell PD-L1 expression and intratumoral immunosuppression. Nat. Commun 5, 5241 (2014). [PubMed: 25348003]

240. Thar Min AK et al. Epithelial-mesenchymal transition-converted tumor cells can induce T-cell apoptosis through upregulation of programmed death ligand 1 expression in esophageal squamous cell carcinoma. Cancer Med. 7, 3321-3330 (2018).

241. Chen L et al. PD-L1 expression promotes epithelial to mesenchymal transition in human esophageal cancer. Cell Physiol. Biochem. 42, 2267-2280 (2017). [PubMed: 28848143]

242. Qiu X et al. PD-L1 confers glioblastoma multiforme malignancy via Ras binding and Ras/Erk/EMT activation. Biochim. Biophys. Acta 1864, 1754-1769 (2018).

243. Fei Z et al. PD-L1 induces epithelial-mesenchymal transition in nasopharyngeal carcinoma cells through activation of the P13K/AKT pathway. Oncol. Res. 27, 801-807 (2019). [PubMed: 30982497]

244. Imai D et al. IFN-y promotes epithelial-mesenchymal transition and the expression of PD-L1 in pancreatic cancer. J. Surg. Res. 240, 115-123 (2019). [PubMed: 30927618]

245. Ren T et al. Osteosarcoma cell intrinsic PD-L2 signals promote invasion and metastasis via the RhoA-ROCK-LIMK2 and autophagy pathways. Cell Death Dis. 10, 261 (2019). [PubMed: 30886151]

246. Kim S et al. PD-L1 expression is associated with epithelial-to-mesenchymal transition in adenocarcinoma of the lung. Hum. Pathol. 58, 7-14 (2016). [PubMed: 27473266]

247. Lou Y et al. Epithelial-mesenchymal transition is associated with a distinct tumor microenvironment including elevation of inflammatory signals and multiple immune checkpoints in lung adenocarcinoma. Clin. Cancer Res. 22, 3630-3642 (2016). [PubMed: 26851185]

248. Critelli R et al. Microenvironment inflammatory infiltrate drives growth speed and outcome of hepatocellular carcinoma: a prospective clinical study. Cell Death Dis. 8, e3017 (2017). [PubMed: 28837142]

249. McNiel EA \& Tsichlis PN Analyses of publicly available genomics resources define FGF-2expressing bladder carcinomas as EMT-prone, proliferative tumors with low mutation rates and high expression of CTLA-4, PD-1 and PD-L1. Signal Transduct. Target Ther 2, 16045 (2017). [PubMed: 28515962]

250. Shrestha $\mathrm{R}$ et al. Monitoring immune checkpoint regulators as predictive biomarkers in hepatocellular carcinoma. Front. Oncol. 8, 269 (2018). [PubMed: 30057891]

251. Shimoji $\mathrm{M}$ et al. Clinical and pathologic features of lung cancer expressing programmed cell death ligand 1 (PD-L1). Lung Cancer 98, 69-75 (2016). [PubMed: 27393509] 
252. Funaki S et al. The prognostic impact of programmed cell death 1 and its ligand and the correlation with epithelial-mesenchymal transition in thymic carcinoma. Cancer Med. 8, 216-226 (2019). [PubMed: 30600651]

253. Wang Y et al. EGFR activation induced Snail-dependent EMT and myc-dependent PD-L1 in human salivary adenoid cystic carcinoma cells. Cell Cycle 17, 1457-1470 (2018). [PubMed: 29954240]

254. Wang L et al. EMT-and stroma-related gene expression and resistance to PD-1 blockade in urothelial cancer. Nat. Commun 9, 3503 (2018). [PubMed: 30158554]

255. Xia Y, Shen S \& Verma IM NF- $\kappa B$, an active player in human cancers. Cancer Immunol. Res. 2, 823-830 (2014). [PubMed: 25187272]

256. Yao-Borengasser A et al. Adipocyte hypoxia promotes epithelial-mesenchymal transition-related gene expression and estrogen receptor-negative phenotype in breast cancer cells. Oncol. Rep. 33, 2689-2694 (2015). [PubMed: 25823469]

257. Peng DH et al. ZEB1 induces LOXL2-mediated collagen stabilization and deposition in the extracellular matrix to drive lung cancer invasion and metastasis. Oncogene 36, 1925-1938 (2017). [PubMed: 27694892]

258. Ingthorsson S, Briem E, Bergthorsson JT \& Gudjonsson T Epithelial plasticity during human breast morphogenesis and cancer progression. J. Mammary Gland. Biol. Neoplasia 21, 139-148 (2016). [PubMed: 27815674]

259. Fantozzi A et al. VEGF-mediated angiogenesis links EMT-induced cancer stemness to tumor initiation. Cancer Res. 74, 1566-1575 (2014). [PubMed: 24413534]

260. Bao S et al. Glioma stem cells promote radioresistance by preferential activation of the DNA damage response. Nature 444, 756-760 (2006). [PubMed: 17051156]

261. Yin H \& Glass J The phenotypic radiation resistance of CD44+/CD24-orlow breast cancer cells is mediated through the enhanced activation of ATM signaling. PLOS ONE 6, e24080 (2011).

[PubMed: 21935375]

262. Chen WJ et al. Multidrug resistance in breast cancer cells during epithelial-mesenchymal transition is modulated by breast cancer resistant protein. Chin. J. Cancer 29, 151-157 (2010). [PubMed: 20109342]

263. Li W et al. Overexpression of Snail accelerates adriamycin induction of multidrug resistance in breast cancer cells. Asian Pac. J. Cancer Prev. 12, 2575-2580 (2011). [PubMed: 22320957]

264. Saxena M, Stephens MA, Pathak H \& Rangarajan A Transcription factors that mediate epithelialmesenchymal transition lead to multidrug resistance by upregulating ABC transporters. Cell Death Dis. 2, e179 (2011). [PubMed: 21734725]

265. Sun L et al. Novel cancer stem cell targets during epithelial to mesenchymal transition in PTENdeficient trastuzumab-resistant breast cancer. Oncotarget 7, 51408-51422 (2016). [PubMed: 27285982]

266. Bendell JC et al. Central nervous system metastases in women who receive trastuzumab-based therapy for metastatic breast carcinoma. Cancer 97, 2972-2977 (2003). [PubMed: 12784331]

267. Gilles $\mathrm{C}$ et al. Vimentin expression in cervical carcinomas: association with invasive and migratory potential. J. Pathol. 180, 175-180 (1996). [PubMed: 8976877]

268. Stark TW et al. Predictive value of epithelial-mesenchymal-transition (EMT) signature and PARP-1 in prostate cancer radioresistance. Prostate 77, 1583-1591 (2017). [PubMed: 29063620]

269. Creighton CJ, Chang JC \& Rosen JM Epithelial-mesenchymal transition (EMT) in tumorinitiating cells and its clinical implications in breast cancer. J. Mammary Gland. Biol. Neoplasia 15, 253-260 (2010). [PubMed: 20354771]

270. Mak MP et al. A patient-derived, pan-cancer EMT signature identifies global molecular alterations and immune target enrichment following epithelial-to-mesenchymal transition. Clin. Cancer Res. 22, 609-620 (2016). [PubMed: 26420858]

271. Rokavec M, Kaller M, Horst D \& Hermeking H Pan-cancer EMT-signature identifies RBM47 down-regulation during colorectal cancer progression. Sci. Rep. 7, 4687 (2017). [PubMed: 28680090] 
272. Payne RE et al. Viable circulating tumour cell detection using multiplex RNA in situ hybridisation predicts progression-free survival in metastatic breast cancer patients. Br. J. Cancer 106, 1790-1797 (2012). [PubMed: 22538972]

273. Decalf J, Albert ML \& Ziai J New tools for pathology: a user's review of a highly multiplexed method for in situ analysis of protein and RNA expression in tissue. J. Pathol. 247, 650-661 (2019). [PubMed: 30570141] 


\section{Box 1 |}

\section{EMT lineage-tracing strategies in genetically engineered mouse models}

Different strategies have been developed to trace epithelial-mesenchymal transition (EMT) in vivo, addressing three major challenges, including distinguishing post-EMT tumour cells from tumour-associated stromal cells that also exhibit mesenchymal phenotypes, the identification of post-EMT tumour cells that might have reversed back to an epithelial phenotype, and reporting on the dynamic EMT status or partial EMT phenotype of tumour cells. Multiple genetically engineered mouse models have been applied in these EMT lineage-tracing strategies (as depicted below), each of which have a number of advantages and disadvantages.

\section{Tumour-specific fluorescent markers}

Tumour-specific promoter (Tum Pro)-driven Cre is crossed with ubiquitous promoter (Ubi Pro)-driven fluorescence reporter alleles $33,40,49,148,188,224$.

\section{Marked genes}

- $\quad P d \times 1$-Cre (pancreatic ductal adenocarcinoma) $)^{149}$

- $\quad$ MMTV-Cre (breast cancer) ${ }^{33,49}$

- Wap-Cre (breast cancer) ${ }^{49}$

- $\quad$ Krt14-CreER (skin cancer) $)^{224}$

- $\quad$ Lgr5-CreER (skin cancer) 40,224

\section{Advantages}

- Distinguishes EMT tumour cells from tumour-associated stromal cells that exhibit mesenchymal phenotypes

\section{Disadvantages}

- Evaluation of EMT status relies on the analysis of EMT marker expression

- $\quad$ Lgr5-Cre might also be activated in non-epithelial lineages in the Lgr5-CreER skin cancer model

\section{Mesenchymal-specific fluorescent markers}

Mesenchymal-specific promoter (Mes Pro)-driven Cre is crossed with a Ubi Pro-driven fluorescent marker switch model ${ }^{34,49,96,223}$.

\section{Marked genes}

- $\quad$ Fsp1-Cre (breast cancer and pancreatic ductal adenocarcinoma) $34,49,97,225$

- $\quad$ aSMA-Cre (pancreatic ductal adenocarcinoma) ${ }^{225}$

- Vimentin-CreER (breast cancer) ${ }^{34}$ 


\section{Advantages}

- Distinguishes tumour cells that have undergone a cycle of EMT and mesenchymal-epithelial transition (MET) from those that persist with an epithelial phenotype

\section{Disadvantages}

- Only one EMT-related marker can be analysed at a time

- Irreversible; can only trace one-time activation of the EMT programme

- Tracks only the transcriptional regulation of the mesenchymal marker

- Relies on sufficiently strong expression of the mesenchymal marker

\section{Fluorescence-tagged EMT marker genes}

EMT marker gene promoter (EMT Pro; for example, Cdh1, Snail1 or Snail2) is used to directly drive fluorescence expression 226,227 .

\section{Marked genes}

- $\quad$ E-cadherin-CFP (pancreatic ductal adenocarcinoma) $)^{227}$

- $\quad$ Snail-GFP (breast cancer) ${ }^{226}$

- $\quad$ Slug-GFP (breast cancer) ${ }^{226}$

\section{Advantages}

- $\quad$ Reports dynamic changes in EMT status and the 'partial EMT' phenotype during tumour progression

\section{Disadvantages}

- Additional markers are required to distinguish tumour cells from neighbouring stromal cells

- $\quad$ Post-EMT events could be reported as negative if cells reversed back to epithelial phenotype

\section{Clonal lineage-tracing}

A lineage-specific promoter (Lin Pro) is used to induce the random recombination of fluorescent markers (such as Krt5 and Elf5 for the basal and luminal lineages, respectively) ${ }^{50}$.

\section{Marked genes}

- $\quad K r t 5$-rtTA/TetO-cre (breast cancer, basal origin) $)^{50}$

- $\quad$ EIf5-rtTA/TetO-cre (breast cancer, luminal origin) ${ }^{50}$

\section{Advantages}

- Reports the origin and clonal evolution of tumour cells 


\section{Disadvantages}

- $\quad$ Elucidation of EMT status requires additional analyses of EMT marker expression

- Tracing limit on the number of tumour cell colonies

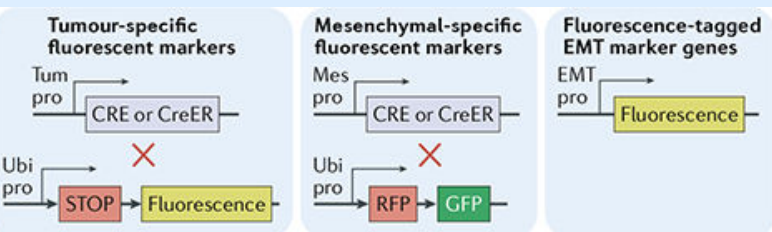

Clonal lineage tracing

Lin

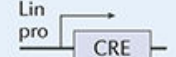

Ubi $\longrightarrow X$

$\stackrel{\text { pro } \underset{\text { STOP }}{\longrightarrow} \rightarrow \text { Fluorescence }}{\longrightarrow}$

$\stackrel{\text { pro }}{\longrightarrow}$ STOP $\rightarrow$ Confetti 


\section{Box 2 |}

\section{Alternatives to intrinsic EMP in metastasis}

Several alternatives to the role of intrinsic epithelial-mesenchymal plasticity (EMP) in metastasis have been proposed, including phenotypic, genotypic, heterotypic and intercellular cooperativity as well as other putative mechanisms that enable mesenchymally shifted tumour cells and/or other cell types to facilitate epithelial tumour cell dissemination.

\section{Phenotypic and genotypic cooperativity}

Cooperativity between epithelial and mesenchymal tumour cell populations has been reported to drive metastasis in multiple cancer models.

In a hamster model of oral carcinoma established from epithelial and/or mesenchymal tumour cells, tumours from the mesenchymal variant were enriched for local invasion, yet tumours established from the mesenchymal or epithelial variants alone could not form metastases $^{228}$. Co-inoculation of both epithelial and mesenchymal variants, either subcutaneously or intravenously, led to metastasis of the epithelial variant.

In a PC-3 prostate cancer tumour xenograft mouse model, co-inoculation of mesenchymal PC-3 variants promoted metastasis of epithelial PC-3 variants ${ }^{126}$.

In a xenograft mouse model of breast cancer, co-injection of epithelial-mesenchymal transition (EMT)-induced HMLER cells (through enforced expression of EMT-activating transcription factor genes such as TWIST1, SNAI1 or SIX1) promoted the metastasis of non-EMT-induced HMLER cells ${ }^{229}$.

In the mouse mammary tumour virus promoter-driven polyoma middle $\mathrm{T}$ oncogene (MMTV-PyMT) model $^{33}$, the combined presence of both epithelial and mesenchymal populations was observed in the vasculature following orthotopic inoculation of Ecadherin $^{\text {low }}$ cells isolated from a mammary tumour. Although both epithelial and mesenchymal variants reached the lungs, E-cadherin ${ }^{\text {low }}$ cells converted to an Ecadherin ${ }^{\text {hgh }}$ phenotype within one to two cell divisions. Whether the more migratory and/or invasive E-cadherin ${ }^{\text {low }}$ cells cooperated to help E-cadheringh ${ }^{\text {hgh }}$ cells reach the lungs is unclear.

\section{Heterotypic cooperativity}

Heterotypic cooperativity arising from clonal heterogeneity has also been reported to drive metastasis ${ }^{230,231}$, challenging the original dogma that metastases arise from a single tumour cell. Evidence of widespread polyclonality has been observed in metastases ${ }^{109,232,233}$, consistent with polyclonality in earlier stages (such as invasion and circulating tumour cell clusters). These studies do not distinguish epithelial-mesenchymal heterogeneity — rather, they represent heterogeneity in general.

\section{Intercellular cooperativity}


Cues provided by cancer-associated fibroblasts, haematopoietic cells and other cells of the pre-metastatic niche have been reported to drive metastasis ${ }^{139}$, and might also promote the survival of epithelial cells during metastasis formation.

\section{Alternative mechanisms}

Potential mechanisms invoked to enable mesenchymally shifted tumour cells and/or other cell types to assist the spread of epithelial tumour cells include cluster dissemination ${ }^{109}$, heterotypic signalling ${ }^{126,228-231}$ and transfer of components of the mesenchymal transcriptome or proteome to epithelial cells (via exosomes, extracellular secretion or other processes) $)^{104,229,234,235}$. 


\section{Box 3 |}

\section{Immunotherapy and eMP}

Phenotypic shifts along epithelial-mesenchymal axis, particularly epithelialmesenchymal transition (EMT), have been reported to influence the likely success of cancer immunotherapy strategies via altered expression of immunomodulatory factors, an unsurprising finding given the wide range of cellular features that are influenced by epithelial-mesenchymal plasticity (EMP).

\section{eMT increases immune checkpoint expression}

- $\quad$ Co-culture with mesenchymal oral squamous cell carcinoma cells increased programmed cell death 1 ligand 1 (PD-L1) expression on dendritic cells and tumour-associated macrophages, which was blocked by inhibiting antigen presentation; EMT reduced PD-L1 expression on tumour cells ${ }^{236}$.

- $\quad$ Melanomas lacking ESRP1 had increased levels of EMT, programmed cell death 1 (PD-1), PD-L1, PD-L2, cytotoxic T-lymphocyte-associated protein 4 (CTLA-4) and immune cytolytic activity ${ }^{237}$.

- In mouse and human melanoma cells, EMT induction through SNAIL1 expression caused progression by enhancing both invasion and immunosuppression; regulatory $\mathrm{T}$ cells were induced and dendritic cells were inhibited, with increased CTLA-4 and PD-1 expression ${ }^{238}$.

- $\quad$ Activation of ZEB1 or repression of microRNA miR-200 induced EMT in human and murine non-small-cell lung cancer cell lines, leading to upregulation of PD-L1 (a miR-200 target), with consequent CD8+ lymphocyte suppression $^{239}$.

- In oesophageal squamous cell carcinoma cell lines, inhibition of the known EMT suppressor GSK3 $\beta$ induced EMT and upregulated PD-L1 expression; EMT-positive tumour cells could induce T cell apoptosis ${ }^{240}$.

\section{Immune checkpoint expression induces eMT}

- $\quad$ PD-L1 expression promoted EMT in oesophageal cancer cells, and PD-1mediated stimulation of PD-L1 enhanced this effect ${ }^{241}$.

- $\quad$ PD-L1 strongly activated EMT programmes in glioblastoma cells via RAS signalling, resulting in increased proliferation and invasion ${ }^{242}$.

- In nasopharyngeal carcinoma, PD-L1 expression activated EMT via PI3KAKT signalling, leading to invasion and metastasis in vitro and in vivo, respectively 243 .

- $\quad$ EMT correlated with increased PD-L1 expression, decreased CD8 ${ }^{+} \mathrm{T}$ cell numbers and increased $\mathrm{FOXP}^{+}$cell numbers in pancreatic cancer. Both EMT and PD-L1 expression could be stimulated by interferon- $\gamma$ in pancreatic cancer cell lines ${ }^{244}$. 
In osteosarcoma, PD-L2 signalling promoted invasion and migration in vitro and in vivo, respectively, via EMT induction ${ }^{245}$.

\section{Associations between eMT and immune checkpoint expression}

- In lung adenocarcinoma tissues, EMT positively correlated with tumour cell PD-L1 expression and levels of CD8 ${ }^{+}$PD- $1^{+}$lymphocyte infiltrates ${ }^{246}$.

- In lung adenocarcinoma data sets, EMT was co-incident with increased expression of PD-L1, PD-L2, PD-1, TIM3, B7-H3, B and T lymphocyte associated (BTLA) and CTLA-4, along with $\mathrm{CD} 4{ }^{+} \mathrm{FOXP}^{+}$regulatory $\mathrm{T}$ cells ${ }^{247}$.

- $\quad$ EMT was associated with increased PD-1 and PD-L1 expression in tumour cells, increased $\mathrm{FOXP}^{+}$lymphocytic infiltrates, and poor prognosis in hepatocellular carcinoma cases ${ }^{248}$.

- $\quad$ Fibroblast growth factor 2 (FGF2)-overexpressing bladder tumours in the The Cancer Genome Atlas showed increased frequency of EMT and increased expression levels of CTLA-4, PD-1 and PD-L1 ${ }^{249}$.

- In hepatocellular carcinoma, PD-L1 expression in high-risk tumours was closely associated with EMT marker expression and poor survival ${ }^{250}$.

- $\quad$ PD-L1 expression correlated with an EMT phenotype and poor outcomes in lung adenocarcinoma but not in lung squamous cell carcinoma ${ }^{251}$.

- In thymic carcinoma tissues, PD-L1 expression correlated with EMT, and levels of EMT, PD-L1 and transforming growth factor- $\beta$ (TGF $\beta$ ) were all increased by immunotherapy 252 .

- $\quad$ Although epidermal growth factor receptor (EGFR) stimulation in salivary adenoid cystic carcinoma cells induced both EMT and PD-L1 expression, EMT was suppressible by $S N A I 1$ knockdown and PD-L1 by $M Y C$ knockdown, but not the reverse ${ }^{253}$.

\section{EMT modulates immunotherapy efficacy}

- In melanomas with high EMT (as indicated by low ESRP1 expression), immune checkpoint molecule expression was increased with a trend towards improved overall survival ${ }^{237}$.

- Immune suppression was seen after induction of EMT in mouse melanoma cells by SNAIL1 overexpression, and these cells were not responsive to activated dendritic cell immunotherapy in mice; resistance was due to low immune reactivity, as well as resistance to cytolysis, and could be reversed using SNAI1-specific siRNA ${ }^{238}$.

- In patients with metastatic urothelial cancers and high $\mathrm{T}$ cell infiltrates treated with nivolumab, a high mRNA-based EMT signature in tumours corresponded with lower response rates and shorter survival; these tumours might correspond to the immune-excluded phenotype ${ }^{254}$. 


\section{Immune surveillance and metastasis}

Only a very small minority of disseminating cells survive and metastasise, the bulk of which are cleared by the immune system. The increase in EMT in circulating cells could potentially assist dissemination by allowing escape from immune-based killing in the circulation. 


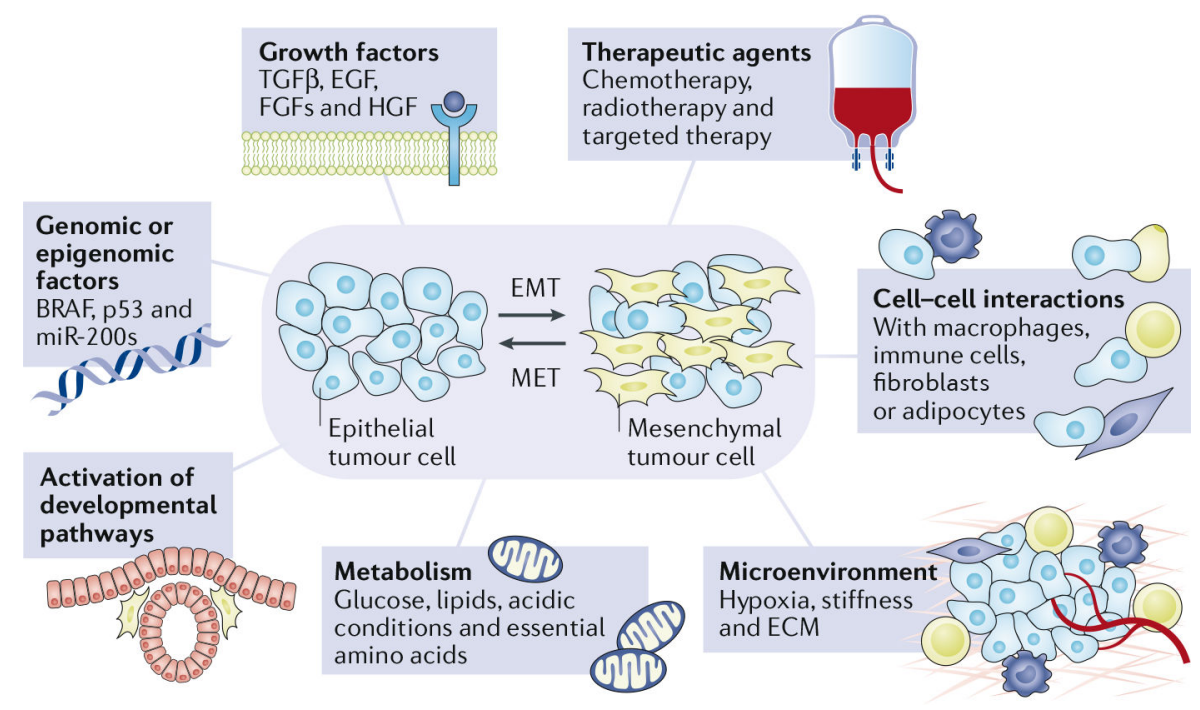

Fig. 1 |. Types of EMP stimuli.

Many categories of factors are known to induce epithelial-mesenchymal transition (EMT), the inhibition or removal of which might promote the reverse process of mesenchymalepithelial transition (MET). Microenvironmental cells (for example, tumour-associated macrophages, hypoxic adipocytes and other inflammatory cells) produce EMT-promoting factors such as transforming growth factor- $\beta$ (TGF $\beta$ ), epidermal growth factor (EGF), fibroblast growth factors (FGFs), hepatocyte growth factor (HGF), tumour necrosis factor, IL-6 (REF. ${ }^{225}$ ) and leptin ${ }^{85,86}$. Through activation of the nuclear factor- $\kappa \mathrm{B}(\mathrm{NF}-\kappa \mathrm{B})$ pathway, these cells invoke crosstalk with EMT-activating transcription factors 255,256 . Alterations of the metabolic microenvironment induced by rapid primary tumour growth might also induce EMT ${ }^{87-90}$, and hypoxia, through the action of hypoxia-inducible factor la (HIF1a), can directly drive the expression of EMT-activating transcription factors in various tumour types ${ }^{51,82,84}$. Matrix stiffness has also been shown to stimulate EMT ${ }^{91,92,257}$. Therapeutic agents have primarily been shown to promote EMT in association with drug resistance ${ }^{43-47,52,70,165-175}$, although some are associated with MET, and these cause significant improvements in disease-free survival and overall survival ${ }^{165}$. Developmental pathways, which might be activated by genomic and/or epigenomic regulators, have also been implicated in epithelial-mesenchymal plasticity (EMP) ${ }^{1,2}$. ECM, extracellular matrix. 


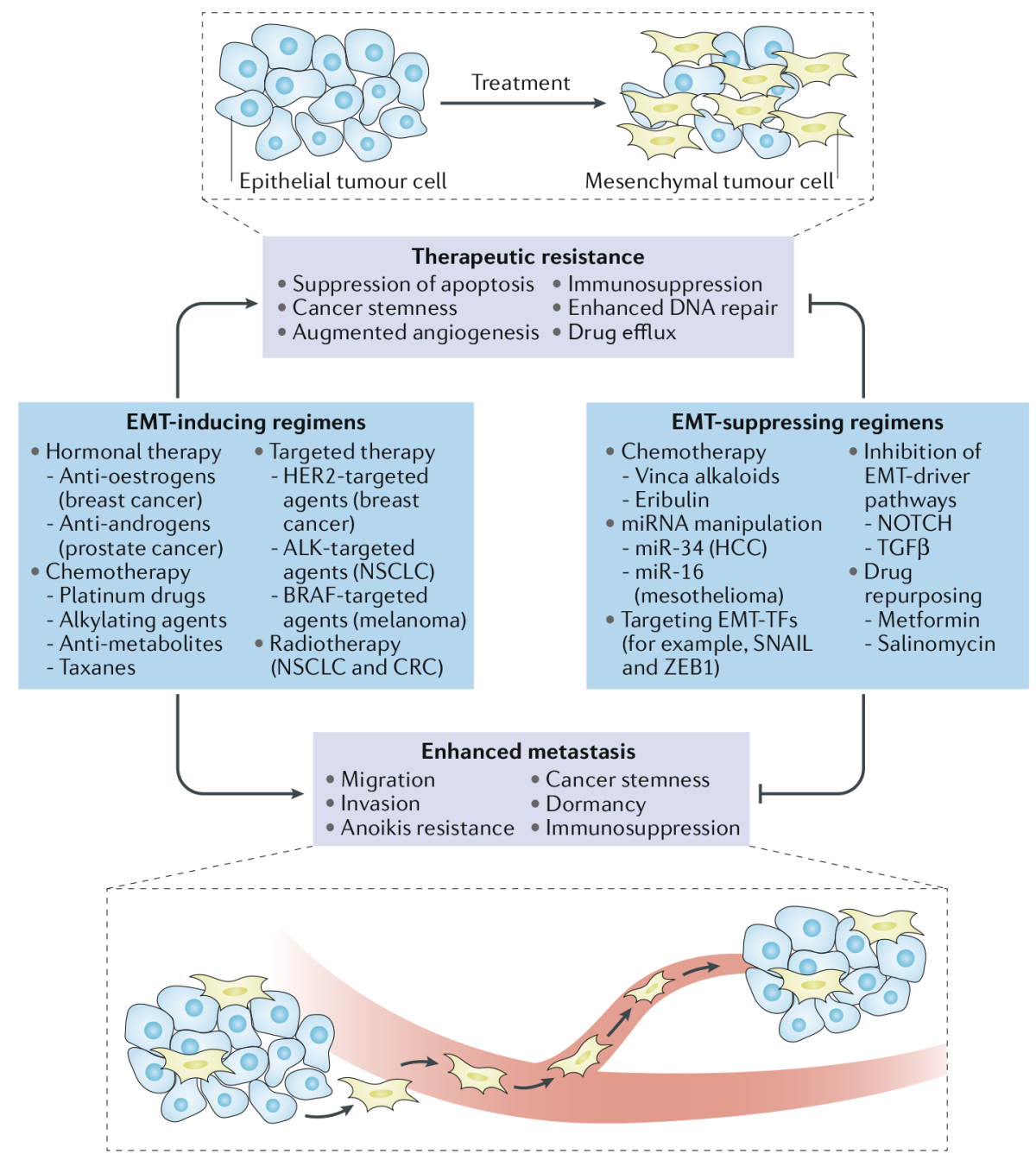

Fig. 2 |. Therapy-induced EMT and potential EMT-suppressing regimens.

Epithelial-mesenchymal transition (EMT) induced by a spectrum of therapeutic agents and modalities has consequences for treatment resistance and/or metastasis across many cancer types. Potential mechanisms through which EMT might contribute to therapeutic resistance include reducing the sensitivity to proapoptotic signals (reviewed in REF ${ }^{258}$ ), acquisition of stemness features ${ }^{66-69}$, stimulation of angiogenesis ${ }^{259}$, upregulating expression of immune checkpoint molecules ${ }^{41}$ and increasing immune suppression by altering the balance of infiltrating immune cells ${ }^{185,237}$, reducing DNA damage in concert with enhancing DNA repair $^{260,261}$, and upregulating expression of export pumps that actively eliminate cytotoxic drugs from cells ${ }^{262-264}$. Furthermore, cells undergoing therapy-induced EMT might proliferate at decreased rates and, therefore, have decreased sensitivity to chemotherapeutic agents $^{34,36,51}$, and migration of cancer cells to a microenvironment that is poorly accessible to drugs (for example, through the blood-brain barrier) might reduce the impact of therapeutic interventions. For example, in human epidermal growth factor receptor 2 (HER2)-positive breast cancer, continued treatment with HER-targeted therapy (for example, trastuzumab) can trigger EMT ${ }^{265}$ and relapse can occur in the brain alone despite an ongoing good response elsewhere in the body ${ }^{266}$. Treatment with existing or novel 
therapies (for example, eribulin or vinca alkaloids) ${ }^{211,212}$ might minimize or revert EMTassociated features and, therefore, reduce the emergence of therapeutic resistance. CRC, colorectal cancer; EMT-TF, EMT-activating transcription factor; HCC, hepatocellular carcinoma; miRNA, microRNA; NSCLC, non-small-cell lung cancer; TGF $\beta$, transforming growth factor- $\beta$. 


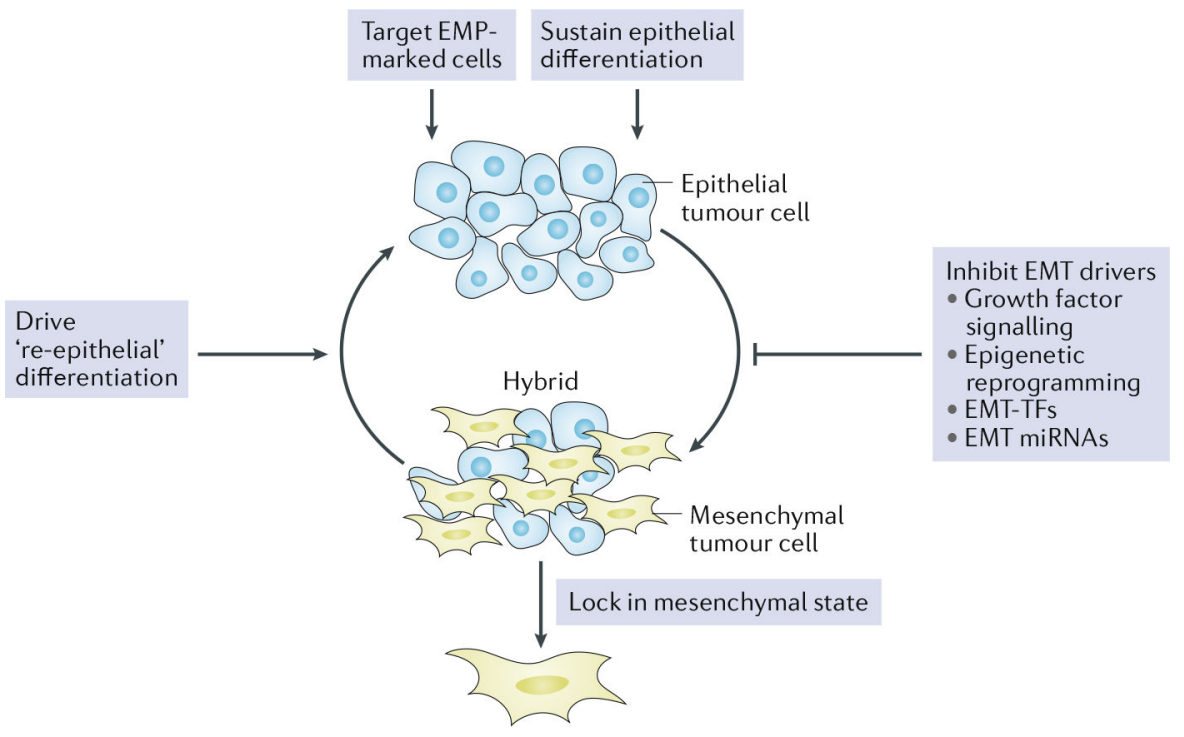

Fig. 3 |. Therapeutic opportunities to address EMP.

The various states produced during epitheLial — mesenchymal plasticity (EMP) provide a number of opportunities to influence cancer progression via different strategies. The supporting rationale for this concept is the notion that targeting or preventing epithelialmesenchymal transition (EMT) to sustain epithelial differentiation or selectively targeting the mesenchymal state by inhibiting EMT-driving targets might be most effective as part of adjuvant therapy for early-stage cancers, where the invasive outgrowth of cells from established deposits as well as quiescent, stem-like, mesenchymally shifted cells that have disseminated could be addressed therapeutically. Late-stage bulky metastases, for which we have very limited effective treatment options, might respond best to therapies that reverse or prevent mesenchymal-epithelial transition (MET) and drive re-epithelial differentiation or selectively target the uniquely EMP-marked epithelial tumour cells that emerge following the EMT-MET cycle process. Further options across the treatment continuum might be to develop therapies that specifically target the unique aspects of the hybrid epithelialmesenchymal phenotype or fix cells in the mesenchymal state to deprive cancers of the progression mechanisms associated with plasticity. EMT-TF, EMT-activating transcription factor; miRNA, microRNA. 
Williams et al.

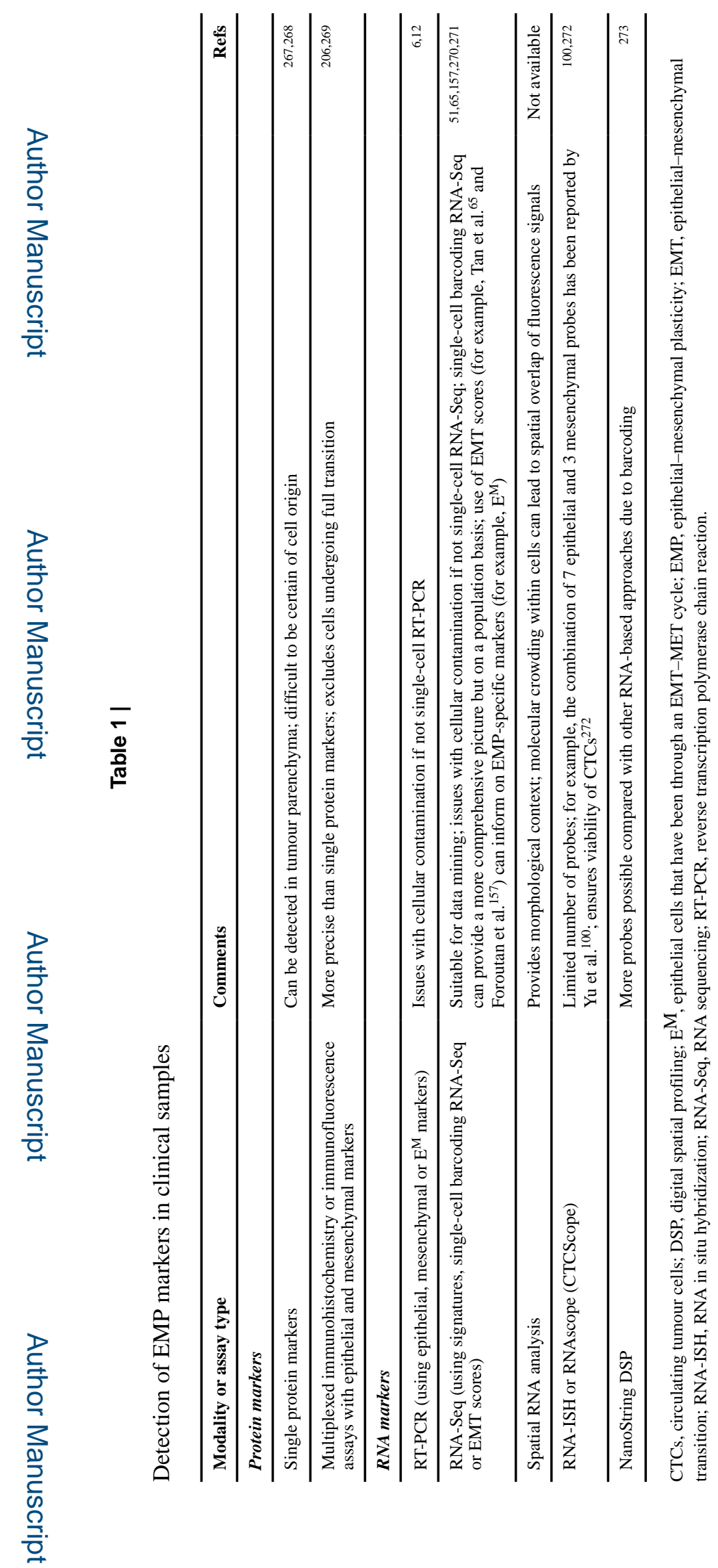

Nat Rev Cancer. Author manuscript; available in PMC 2020 June 01. 